\title{
The complete structure of the large subunit of the mammalian mitochondrial ribosome
}

\section{Journal Article}

\section{Author(s):}

Greber, Basil J.; Boehringer, Daniel; Leibundgut, Marc; Bieri, Philipp; Leitner, Alexander; Schmitz, Nikolaus; Aebersold, Ruedi; Ban, Nenad

\section{Publication date:}

2014-10-01

\section{Permanent link:}

https://doi.org/10.3929/ethz-b-000093239

Rights / license:

In Copyright - Non-Commercial Use Permitted

Originally published in:

Nature 515(7526), https://doi.org/10.1038/nature13895 
Title: The complete structure of the large subunit of the mammalian mitoribosome

Authors: Basil J. Greber ${ }^{1,{ }^{*}}$, Daniel Boehringer ${ }^{1,{ }^{*}}$, Marc Leibundgut ${ }^{1,{ }^{*}}$, Philipp Bieri ${ }^{1}$, Alexander Leitner ${ }^{2}$, Nikolaus Schmitz ${ }^{1}$, Ruedi Aebersold ${ }^{2,3}$, and Nenad $\mathrm{Ban}^{1, \dagger}$

* These authors contributed equally to this work

Affiliations: ${ }^{1}$ Department of Biology, Institute of Molecular Biology and Biophysics, Otto-Stern-Weg 5, ETH Zurich, CH-8093 Zurich, Switzerland ${ }^{2}$ Department of Biology, Institute of Molecular Systems Biology, AugustePiccard-Hof 1, ETH Zurich, CH-8093 Zurich, Switzerland

${ }^{3}$ Faculty of Science, University of Zurich, Zurich, Switzerland

† Correspondence to: ban@mol.biol.ethz.ch (N.B.) 
Summary: Mitochondrial ribosomes (mitoribosomes) are extensively modified ribosomes of bacterial descent specialized for the synthesis and insertion of membrane proteins that are critical for energy conversion and ATP production inside mitochondria ${ }^{1}$. Mammalian mitoribosomes, which are composed of $39 S$ and $28 S$ subunits ${ }^{2}$, have diverged dramatically from the bacterial ribosomes from which they are derived, rendering them unique compared to bacterial, eukaryotic cytosolic, and fungal mitochondrial ribosomes ${ }^{3-5}$. We have previously determined the architecture of the porcine (Sus scrofa) 395 subunit at $4.9 \AA$ resolution ${ }^{6}$, which is highly homologous to the human mitoribosomal large subunit. Here we present the complete atomic structure of the porcine 395 large mitoribosomal subunit determined in the context of a stalled translating mitoribosome at $3.4 \AA$ resolution by cryo-electron microscopy and chemical crosslinking/mass spectrometry. The structure reveals the locations and the detailed folds of 50 mitoribosomal proteins, shows the highly conserved mitoribosomal peptidyl transferase active site in complex with its substrate tRNAs, and defines the path of the nascent chain in mammalian mitoribosomes along their idiosyncratic exit tunnel. Furthermore, we present evidence that a mitochondrial tRNA has become an integral component of the central protuberance of the 395 subunit where it architecturally substitutes for the absence of the 5S rRNA, a ubiquitous component of all cytoplasmic ribosomes. 
Main Text: Our previous analysis of the porcine 39S mitoribosomal large subunit at $4.9 \AA$ resolution showed the overall fold of the mitoribosomal $16 \mathrm{~S}$ rRNA as well as the localization of seven mitoribosomal-specific proteins ${ }^{6}$. However, proteins and protein extensions for which no homology models could be generated could not be modeled at this resolution. Additionally, due to the extensive differences between yeast and mammalian mitoribosomes, the recently reported high-resolution structure of the yeast mitoribosomal large subunit ${ }^{5}$ is of limited use for understanding of the mammalian-specific aspects of mitoribosomal structure and function ${ }^{4,7}$.

Cryo-EM data of porcine 55S mitoribosomes acquired on a movie modeenabled direct electron detector combined with movie frame realignment to compensate for beam-induced specimen motion ${ }^{8}$ and maximum-likelihood based image classification and alignment ${ }^{9}$ yielded a 3D-reconstruction of the $55 \mathrm{~S}$ mitoribosome (Extended Data Fig. 1a, b) at $3.6 \AA$ resolution (FSC $=$ 0.143 , "gold standard"). However, due to differences in local resolution (Extended Data Fig. 1C), the quality of the density in the $28 \mathrm{~S}$ subunit part of the cryo-EM map (28S subunit resolution $4.1 \AA$ ) was of insufficient quality for reliable model building and refinement. Therefore, we focused the refinement on the 39S subunit, resulting in an improved 3D-reconstruction of the $39 \mathrm{~S}$ subunit at $3.4 \AA$ resolution (Extended Data Fig. 2), suitable for de-novo model building, structure refinement, and validation.

We were able to build and refine the atomic coordinates of $95 \%$ of the $16 \mathrm{~S}$ rRNA nucleotides and 50 of the 52 proteins of the $39 S$ subunit (Fig. 1a, b; Extended Data Figs. 3, 4; Supplementary Table 1). The proteins uL1m and $\mathrm{bL} 7 \mathrm{~m} / \mathrm{bL} 12 \mathrm{~m}$ (nomenclature according to ${ }^{10}$ ) form the highly flexible L7/L12 and L1 stalks and are therefore not visible in our maps. The proposed total count of 52 proteins includes mL65 (MRPS30) and mL66 (MRPS18A), previously assigned to the $28 \mathrm{~S}$ small subunit, but now identified as components of the $39 \mathrm{~S}$ subunit (Extended Data Fig 5a-i; Supplementary Discussion; Extended Data Table 1) in addition to 50 of the 51 proteins previously assigned to the $39 \mathrm{~S}$ subunit ${ }^{4,11}$. We found no evidence for the presence of MRPL56 in our mitoribosomal sample by mass-spectrometry 
(Supplementary Table 2). All our protein placements at lower resolution ${ }^{6}$ were confirmed by the high-resolution structure, validating our approach that combined cryo-EM with chemical crosslinking and mass spectrometry (CXMS) ${ }^{12}$.

The mammalian mitoribosome contains 11 additional proteins that are unique to higher eukaryotes in comparison to the yeast $54 \mathrm{~S}$ large mitoribosomal subunit (Extended Data Fig. 4). Several of these proteins localize to functionally important areas, such as the $L 7 / 12$ stalk base and the central protuberance (CP) (Extended Data Fig. 5j-I), while others are located on the solvent-exposed side of the subunit (Fig. 1C, d, Extended Data Fig. 6).

The peptidyl transferase center (PTC) of the ribosome is formed exclusively by rRNA ${ }^{13}$ and catalyzes peptide bond formation ${ }^{14}$. The structural similarity of the rRNA elements in the PTC active site of the mammalian mitoribosome to those in both bacterial and eukaryotic cytosolic ribosomes indicates that the mechanism of the catalytic core of the mammalian mitoribosome is unchanged, consistent with the high level of sequence conservation in this region of the rRNA ${ }^{13,15,16}$ (Fig. 2). In bacterial ribosomes, bL27 is the only protein that approaches the ribosomal active site ${ }^{15}$. The density for the very $\mathrm{N}$-terminus of bL27m, although weak in our cryo-EM map, indicates that bL27m also approaches the PTC. We do not observe any density for additional protein elements of mitoribosomal proteins reaching near the catalytic site.

The $55 \mathrm{~S}$ mitoribosomes used for structure determination were purified partially occupied with A- and P-site tRNAs in the PTC, and the subpopulation obtained by computational sorting (Extended Data Fig. 1) and used for structure determination was enriched with particles containing bound tRNAs. The heterogeneous mixture of tRNA species occupying these mitoribosomes precludes building of any specific tRNA species into the density. However, the universally conserved CCA-3'-ends, which carry the covalently bound amino acid substrates or the nascent chain in the A- and P-site, respectively, are identical in all tRNAs, and could therefore be unambiguously identified and 
built into the cryo-EM density near the PTC (Fig. 2b). Their interactions with the 16S rRNA near the PTC are conserved from the 23S rRNA-tRNA interactions in bacterial ribosomes ${ }^{15}$. The conformation of nucleotide $U 1327$ near the PTC is similar to the conformation of the corresponding U1506 nucleotide observed in the activated state of the bacterial PTC in the presence of properly accommodated PTC substrates ${ }^{15}$, indicating that our tRNA-containing complexes correspond to a functional state of the mitoribosome (Fig. 2c).

The polypeptide exit tunnel in the large ribosomal subunit starts at the PTC and leads through the large subunit to the polypeptide exit site (PES), where the nascent protein leaves the ribosome ${ }^{13,17}$. In bacteria, the polypeptide tunnel is of high medical relevance because it is a binding site for antibiotics ${ }^{18}$, whereas the PES region is critical for the processing, folding, and targeting of nascent polypeptides ${ }^{19}$. Our structure shows that the entry into the mammalian mitoribosomal polypeptide exit tunnel is very similar to the bacterial tunnel, which is the binding site of macrolide antibiotics ${ }^{18}$. However, mammalian mitoribosomes are less susceptible to macrolide antibiotics than bacterial ribosomes ${ }^{20}$. The structure of the large mitoribosomal subunit from yeast indicated that a constriction of the tunnel near the PTC might be the reason for antibiotic specificity ${ }^{5}$. However, such a constriction is not present in mammalian mitoribosomes (Fig. 3a). Therefore, their resistance to macrolide binding is more likely due to the presence of a $\mathrm{G}$ nucleotide at position 1051 (Fig. 3a) of the 16S rRNA (2058 in E. coli 23S rRNA), as is the case for eukaryotic cytosolic and archaeal ribosomes ${ }^{16,21}$. Because of the high homology between human and porcine mitoribosomes and the conservation of $\mathrm{G} 1051$ in the human $16 \mathrm{~S}$ rRNA ${ }^{22}$, this mechanism likely also applies to human mitoribosomes.

In mitoribosomes, the strongly remodeled PES region is an important binding site for the membrane insertion machinery, including the Oxa1L insertase ${ }^{1,23-}$ 25. In our reconstruction of the 395 subunit, we observe a continuous threadlike density inside the polypeptide tunnel extending from the P-site tRNA in the PTC towards the canonical PES, suggesting that we are 
observing stalled ribosomes that carry nascent polypeptide chains (Fig. 3b, c). Two alternative exit sites of the mitoribosomal tunnel have been suggested previously: (i) the tunnel of the mammalian mitoribosome bears a lateral opening, termed the polypeptide accessible site (PAS) ${ }^{3,25}$ and (ii) the tunnel of the yeast $54 \mathrm{~S}$ mitoribosomal large subunit has been suggested to deviate from the otherwise universally conserved path to emanate at a location close to proteins $\mathrm{mL} 44$ and $\mathrm{mL} 50^{5}$. Our data indicate that in mammalian mitoribosomes, the putative exit site observed in yeast mitoribosomes is blocked by the 16S rRNA (Fig. 3d) and that the second proposed lateral exit site, the PAS ${ }^{3}$, is partially blocked by mitoribosomal-specific protein elements (Fig. 3e). Therefore, based on our structural data on the 395 mitoribosomal subunit and the direct observation of the nascent chain features in our cryoEM reconstruction, we consider it most likely that mammalian mitoribosomes use the canonical nascent polypeptide exit site (Fig. 3f).

\section{Based on rRNA sequence analysis and lower-resolution cryo-EM} reconstructions, it has been suggested that the E-site of the mammalian 395 subunit is weak or completely absent ${ }^{3,25}$. Indeed, in contrast to the yeast $54 \mathrm{~S}$ mitoribosomal subunit ${ }^{5}$, our structure does not show density for E-site tRNA and confirms that a number of rRNA elements interacting with the E-site tRNA in bacterial ribosomes, such as H68, are absent (Extended Data Fig. 7a, b). However, our structure reveals that the binding pocket for the CCA-3' end of the E-site tRNA observed in bacteria ${ }^{15,26}$ is well conserved in the 395 subunit and in a similar conformation compared to the occupied bacterial E-site (Extended Data Fig. 7c). This makes it likely that the tRNAs in mammalian mitoribosomes move through the ribosome by binding to the E-site on the $39 \mathrm{~S}$ subunit according to the same mechanistic principles as established for cytoplasmic ribosomes ${ }^{27,28}$.

Even though the basic mechanics of tRNA translocation are likely conserved, the mammalian mitoribosome may support accurate tRNA binding or translocation with mitoribosomal-specific protein elements, such as the P-site finger, which reaches near the P-site tRNA ${ }^{3}$. However, the details of these interactions are still poorly understood. We see EM density for two protein 
elements that approach the area where the tRNAs are located, although at lower local resolution than the surrounding area (Extended Data Fig. 7d-f). At the $\mathrm{CP}, \mathrm{mL} 48$ and $\mathrm{mL} 40$ may form a portion of the P-site finger (Extended Data Fig. 7d), which is inserted between the A- and P-site tRNAs and contacts both tRNAs (Extended Data Fig. 7e, f). Additionally, a long a-helix of mL64 (CRIF1, MRPL59) that emanates from the L1-stalk region of the subunit projects towards the P-site finger and may also contribute to protein-tRNA interactions in this region (Extended Data Fig. 7d).

We have previously demonstrated the presence of a second ribosomal RNA molecule in the CP of the $39 \mathrm{~S}$ subunit in a position similar to a domain of $5 \mathrm{~S}$ rRNA in bacterial ribosomes ${ }^{6}$. The CP is better ordered in the $55 \mathrm{~S}$ mitoribosome due to intersubunit contacts to the head domain of the $28 \mathrm{~S}$ subunit that likely involve $m L 46$ and $m L 48$ (Extended Data Fig. 5j, k), revealing that the RNA density has the characteristic L-shape of a tRNA (Fig. 4a). Without any prior assumptions regarding the identity of this RNA, we built the sequence of purines and pyrimidines into the better-resolved part of the cryo-EM density in the area tentatively assigned as the anticodon stem of the tRNA (Extended Data Fig. 8a-e). Alignment of this pattern to the porcine mitochondrial genome as well as the sequences of all cytosolic tRNAs and rRNAs identified mitochondrial tRNA ${ }^{\text {Phe }}$ as the most likely candidate ${ }^{29,30}$. We therefore conclude that a tRNA, likely mitochondrial tRNA ${ }^{\text {Phe }}$, is present in the CP of the 39S subunit. We refer to it as CP tRNA to distinguish it from tRNAs used for decoding in the intersubunit space.

The CP tRNA acts as an organizing scaffold for the 39S CP by forming extensive contacts to the surrounding proteins $\mathrm{uL} 18 \mathrm{~m}, \mathrm{~mL} 38, \mathrm{~mL} 40$, and $\mathrm{mL} 48$ (Fig. 4b). Prokaryotic uL18 binds to domain $\beta$ of the $5 \mathrm{~S} \mathrm{rRNA}{ }^{13}$, while mitochondrial uL $18 \mathrm{~m}$ binds the CP tRNA near the anticodon stem loop in mammalian mitoribosomes (Fig. 4b). uL18 is absent from the yeast mitoribosome, which lacks both 5S rRNA and CP tRNA, but contains an rRNA expansion segment in this area ${ }^{5}$. Other elements of mitoribosomal $\mathrm{CP}$ architecture, such as $\mathrm{mL} 38, \mathrm{~mL} 40$, and $\mathrm{mL} 46$, are conserved between yeast and mammals in spite of these differences in RNA content and structure 
(Extended Data Fig. 8g-I). The recruitment of an entirely novel RNA molecule highlights the extreme evolutionary plasticity of the mammalian mitoribosome. The high-resolution structure presented here enables future structure-based experiments and paves the way to a detailed molecular understanding of mitoribosomal function and evolution (Supplementary Discussion; Extended Data Fig. 9).

Online Content Methods, Extended Data display items and Source Data are available in the online version of the paper; references unique to these sections appear only in the online paper.

\section{References}

1 Ott, M. \& Herrmann, J. M. Co-translational membrane insertion of mitochondrially encoded proteins. Biochim. Biophys. Acta 1803, 767775 (2010).

2 O'Brien, T. W. The general occurrence of $55 \mathrm{~S}$ ribosomes in mammalian liver mitochondria. J. Biol. Chem. 246, 3409-3417 (1971).

3 Sharma, M. R. et al. Structure of the mammalian mitochondrial ribosome reveals an expanded functional role for its component proteins. Cell 115, 97-108 (2003).

4 Desmond, E., Brochier-Armanet, C., Forterre, P. \& Gribaldo, S. On the last common ancestor and early evolution of eukaryotes: reconstructing the history of mitochondrial ribosomes. Res. Microbiol. 162, 53-70 (2011).

5 Amunts, A. et al. Structure of the yeast mitochondrial large ribosomal subunit. Science 343, 1485-1489 (2014).

6 Greber, B. J. et al. Architecture of the large subunit of the mammalian mitochondrial ribosome. Nature 505, 515-519 (2014).

7 Smits, P., Smeitink, J. A. M., van den Heuvel, L. P., Huynen, M. A. \& Ettema, T. J. G. Reconstructing the evolution of the mitochondrial ribosomal proteome. Nucleic Acids Res. 35, 4686-4703 (2007).

$8 \mathrm{Li}, \mathrm{X}$. et al. Electron counting and beam-induced motion correction enable near-atomic-resolution single-particle cryo-EM. Nat. Meth. 10, 584-590 (2013).

9 Scheres, S. H. W. RELION: Implementation of a Bayesian approach to cryo-EM structure determination. J. Struct. Biol. 180, 519-530 (2012).

10 Ban, N. et al. A new system for naming ribosomal proteins. Curr. Opin. Struct. Biol. 24, 165-169 (2014).

11 Koc, E. C. et al. Identification and characterization of $\mathrm{CHCHD1,}$ AURKAIP1, and CRIF1 as new members of the mammalian mitochondrial ribosome. Front. Physiol. 4, 183 (2013). 
12 Walzthoeni, T., Leitner, A., Stengel, F. \& Aebersold, R. Mass spectrometry supported determination of protein complex structure. Curr. Opin. Struct. Biol. 23, 252-260 (2013).

13 Ban, N., Nissen, P., Hansen, J., Moore, P. B. \& Steitz, T. A. The complete atomic structure of the large ribosomal subunit at $2.4 \mathrm{~A}$ resolution. Science 289, 905-920 (2000).

14 Beringer, M. \& Rodnina, M. V. The ribosomal peptidyl transferase. Mol. Cell 26, 311-321 (2007).

15 Voorhees, R. M., Weixlbaumer, A., Loakes, D., Kelley, A. C. \& Ramakrishnan, $\mathrm{V}$. Insights into substrate stabilization from snapshots of the peptidyl transferase center of the intact 70 S ribosome. Nat. Struct. Mol. Biol. 16, 528-533 (2009).

16 Klinge, S., Voigts-Hoffmann, F., Leibundgut, M., Arpagaus, S. \& Ban, N. Crystal structure of the eukaryotic $60 \mathrm{~S}$ ribosomal subunit in complex with initiation factor 6. Science 334, 941-948 (2011).

17 Voss, N. R., Gerstein, M., Steitz, T. A. \& Moore, P. B. The geometry of the ribosomal polypeptide exit tunnel. J. Mol. Biol. 360, 893-906 (2006).

18 Schlünzen, F. et al. Structural basis for the interaction of antibiotics with the peptidyl transferase centre in eubacteria. Nature 413, 814-821 (2001).

19 Boehringer, D., Greber, B. \& Ban, N. in Ribosome Structure, Function, and Dynamics (eds Rodnina, M., Wintermeyer, W. \& Green, R.) 200212 (Springer, 2011).

20 Zhang, L. et al. Antibiotic susceptibility of mammalian mitochondrial translation. FEBS Lett. 579, 6423-6427 (2005).

21 Tu, D., Blaha, G., Moore, P. B. \& Steitz, T. A. Structures of MLSBK antibiotics bound to mutated large ribosomal subunits provide a structural explanation for resistance. Cell 121, 257-270 (2005).

22 Cannone, J. J. et al. The comparative RNA web (CRW) site: an online database of comparative sequence and structure information for ribosomal, intron, and other RNAs. BMC Bioinformatics 3, 2 (2002).

23 Stiburek, L. et al. Knockdown of human Oxa1l impairs the biogenesis of F1Fo-ATP synthase and NADH:ubiquinone oxidoreductase. J. Mol. Biol. 374, 506-516 (2007).

24 Saller, M. J., Wu, Z. C., de Keyzer, J. \& Driessen, A. J. M. The YidC/Oxa1/Alb3 protein family: common principles and distinct features. Biol. Chem. 393, 1279-1290 (2012).

25 Agrawal, R. K. \& Sharma, M. R. Structural aspects of mitochondrial translational apparatus. Curr. Opin. Struct. Biol. 22, 797-803 (2012).

26 Selmer, M. et al. Structure of the 70 S ribosome complexed with mRNA and tRNA. Science 313, 1935-1942 (2006).

27 Moazed, D. \& Noller, H. F. Intermediate states in the movement of transfer RNA in the ribosome. Nature 342, 142-148 (1989).

28 Agirrezabala, X. et al. Visualization of the hybrid state of tRNA binding promoted by spontaneous ratcheting of the ribosome. Mol. Cell 32 , 190-197 (2008).

29 Jühling, F. et al. tRNAdb 2009: compilation of tRNA sequences and tRNA genes. Nucleic Acids Res. 37, D159-162 (2009).

30 Helm, M. et al. Search for characteristic structural features of mammalian mitochondrial tRNAs. RNA 6, 1356-1379 (2000). 
Supplementary Information is linked to the online version of the paper at www.nature.com/nature.

Acknowledgments Cryo-EM data was collected at the electron microscopy facility of ETH Zurich (ScopeM). We thank Peter Tittmann (ScopeM) for support. We thank Jian Yu and Constance Ciaudo for discussions. This work was supported by the Swiss National Science Foundation (SNSF), the National Center of Excellence in Research (NCCR) Structural Biology program of the Swiss National Science Foundation (SNSF), European Research Council (ERC) grant 250071 under the European Community's Seventh Framework Programme (to N.B.), the Commission of the European Communities through the PROSPECTS consortium (EU FP7 projects 201648, 233226) (R.A.), and the European Research Council (ERC-2008-AdG 233226) (R.A.).

Author Contributions P.B. and B.J.G. performed preparation of the mitoribosomes. B.J.G., P.B., and D.B. prepared cryo-EM samples. D.B. and B.J.G. acquired the cryo-EM data. B.J.G. and P.B. calculated the cryo-EM reconstructions with support from D.B.. M.L. and B.J.G. built the atomic model. M.L, N.S, and D.B. performed coordinate refinement of the atomic model. M.L., B.J.G., P.B., D.B., and N.B. interpreted the structure. A.L. performed CX-MS experiments in the laboratory of R.A. All authors contributed to the final version of the paper.

Author Information The $3.4 \AA$ cryo-EM map of the 395 mitoribosomal subunit has been deposited in the Electron Microscopy Databank with accession code EMD-2787. The coordinates of the atomic structure of the 395 mitoribosomal subunit have been deposited in the protein databank with PDB codes $4 \mathrm{v} 1 \mathrm{a}$ and $4 \mathrm{v} 19$. Reprints and permissions information is available at www.nature.com/reprints. ETH Zürich has filed a patent application to use the coordinates of the $39 \mathrm{~S}$ mitoribosomal subunit for development of compounds that can (i) specifically bind to the mitochondrial ribosome and 
interfere with mitochondrial translation or (ii) specifically inhibit translation of pathogenic organisms without interfering with mitochondrial translation. Correspondence and requests for materials should be addressed to N.B. (ban@mol.biol.ethz.ch). 


\section{METHODS}

\section{Sample preparation and data acquisition}

Porcine mitochondria and mitochondrial ribosomes were prepared as described ${ }^{6}$. Cryo-EM grids were prepared by applying a sample solution containing $45 \mathrm{nM} 55 \mathrm{~S}$ mitoribosomes in sample buffer $(20 \mathrm{mM}$ Hepes- $\mathrm{KOH}$ $\mathrm{pH} 7.4,40 \mathrm{mM} \mathrm{MgCl}_{2}, 50 \mathrm{mM} \mathrm{KCl}, 1 \mathrm{mM}$ DTT) to Quantifoil R2/2 holey carbon grids (Quantifoil Micro Tools) coated with a thin carbon film (prepared using a Balzers BAE 120 thin film coating system). The grids were manually blotted with damp filter paper from the front and manually plunged into a 2:3 mixture of ethane and propane ${ }^{31}$ at liquid nitrogen temperature. Cryo-EM data was collected using a Titan Krios cryo-transmission electron microscope (FEI Company) operated at $300 \mathrm{keV}$ equipped with a Falcon II direct electron detector (FEI Company) at a magnification of 100'000 x (accuracy of magnification calibration approx. $0.5 \%$ ) and defocus values of -0.8 to -3.0 $\mu \mathrm{m}$. The EPU software (FEI Company) was used for automated data acquisition. Images were acquired by collecting 7 movie frames (combined dose 20 electrons $/ \AA^{2}$ ) per exposure (combined exposure time $0.77 \mathrm{~s}$ ) after discarding the first frame (55 ms). The movie frames were subsequently aligned to correct for beam-induced specimen motion using DOSEFGPU DRIFTCORR ${ }^{8}$.

\section{Structure calculation}

Data was acquired in two sessions, yielding two datasets that were initially processed independently. Overview images of the holes from which data had been collected were acquired by the EPU software and inspected to discard areas of poor ice quality or cracks in the carbon film. From the drift-corrected micrographs, CTF parameters were estimated using CTFFIND ${ }^{32}$ from within RELION ${ }^{9}$. The quality of the power spectra produced in this step was evaluated and poor quality micrographs were rejected. Roughly 231'000 (dataset 1) and 328'000 (dataset 2) particles were selected using Batchboxer (EMAN 1.9) ${ }^{33}$ as described ${ }^{6}$. Further image processing was performed in 
RELION $1.2^{9}$. After an initial round of 2D-classification with 120 classes at a pixel size of $5.6 \AA$ on the object scale (80 pixel frames) to remove most of the non-ribosomal particles and 39S subunits, a 3D-classification with 10 classes at the same pixel size was performed, using a low-pass filtered reconstruction of the $55 \mathrm{~S}$ mitoribosome as an initial reference ${ }^{6}$, to sort the particle images into homogeneous subpopulations and again remove particles that were not $55 \mathrm{~S}$ mitoribosomes (Extended Data Fig. 1a). After pooling of the 55S mitoribosomal particles from the two datasets, a second round of $3 \mathrm{D}$ classification with 10 classes was performed. Roughly 142'000 particles were then selected and refined to high resolution using "gold standard" 34 refinement in RELION. A 3.6 A resolution reconstruction of the 55S mitoribosome was obtained (Extended Data Fig. 1b, c), which however was not of sufficient resolution for de-novo model building of the $28 \mathrm{~S}$ subunit part due to low local resolution ( $4.1 \AA$ on average for the $28 \mathrm{~S}$ subunit). Therefore, refinement was limited to the 395 subunit by applying a mask around the subunit as described previously ${ }^{5,35}$, resulting in a $3.4 \AA$ resolution reconstruction of the $39 \mathrm{~S}$ subunit (Extended Data Fig. 2) that could be used for manual atomic model building, refinement, and validation.

\section{Chemical crosslinking using S. scrofa 55 S mitoribosomes}

Crosslinking using the amine-reactive disuccinimidyl suberate (DSS) was performed as described previously ${ }^{6}$ using $75 \mu \mathrm{g}$ of the $55 \mathrm{~S}$ complex at a concentration of $0.5 \mathrm{mg} / \mathrm{mL}$. Combined crosslinking of acidic residues and zero-length crosslinking using pimelic acid dihydrazide (PDH) and 4-(4,6dimethoxy-1,3,5-triazin-2-yl)-4-methylmorpholinium chloride (DMTMM) was performed following the procedure described recently ${ }^{36}$. The ribosome sample $(75 \mu \mathrm{g})$ was prepared at a concentration of $0.5 \mathrm{mg} / \mathrm{mL}$ in crosslinking buffer (20mM HEPES-KOH pH 7.6, $100 \mathrm{mM} \mathrm{KCl}, 20 \mathrm{mM} \mathrm{MgCl}_{2}, 1 \mathrm{mM}$ DTT, $10 \%(\mathrm{w} / \mathrm{v})$ sucrose). PDH was added as an equimolar mixture of isotopically light $\left(d_{0}\right)$ and heavy $\left(d_{10}\right)$ form (both obtained from Sigma-Aldrich) to a final concentration of $9 \mathrm{mg} / \mathrm{mL}$. The crosslinking reaction was initiated by addition of DMTMM to a final concentration of $12 \mathrm{mg} / \mathrm{mL}$, and the sample was incubated at $37^{\circ} \mathrm{C}$ for $45 \mathrm{~min}$. The reaction was stopped by gel filtration using 
a Zeba spin column (ThermoFisher/Pierce) according to the manufacturer's instructions.

Further sample treatment (reduction, alkylation, enzymatic digestion using endoproteinase Lys-C and trypsin, size exclusion chromatography fractionation) and liquid chromatography-tandem mass spectrometry (LC-MS) analysis (Easy nLC-1000 / Orbitrap Elite, ThermoFisher) followed published procedures ${ }^{6,37,38}$.

MS data were searched using $x$ Quest/xProphet ${ }^{39}$ as described previously ${ }^{6,36}$ against a database compiled from $S$. scrofa mitoribosomal protein sequences identified by protein-protein BLAST ${ }^{40}$ or BLAST searches of human mitoribosomal protein sequences against a translated nucleotide database (NCBI nucleotide collection, http://blast.ncbi.nlm.nih.gov/; limited to S. scrofa sequences), as described ${ }^{6}$. A false discovery rate of $5 \%$ was set using xProphet, independently for different crosslinking chemistries and for intraand inter-protein crosslinks.

\section{Analysis of non-crosslinked S. scrofa 55S ribosomes using LC-MS/MS.}

For the assignment of mitoribosomal protein variants (Supplementary Table 2) and label-free relative quantitation of $55 \mathrm{~S}$ subunits (Extended Data Fig. $5 \mathrm{i}$ ), a $10 \mu \mathrm{g}$ aliquot of untreated $55 \mathrm{~S}$ mitoribosomes was reduced, alkylated and digested with Lys-C and trypsin. $1 \mu \mathrm{g}$ of the digest was analyzed in duplicate by LC-MS/MS using the same experimental set-up as for the crosslinked fractions, with the following exceptions: a 120 min gradient from 5 to $30 \% \mathrm{~B}$, where solvent A water/acetonitrile/formic acid (95:5:0.1 (v/v/v)) and B acetonitrile/water/formic acid (95:5:0.1 (v/v/v)), was used, and the 15 most abundant precursors with charge states of $2+$ or above were selected for fragmentation.

MS data were converted into the Mascot generic format using MzXML2Search (part of the Trans-Proteomic pipeline ${ }^{41}$ ) and searched against the custom mitoribosomal protein database (see above) using Mascot 
2.4.01 ${ }^{42}$. Protein grouping was performed in Mascot as described in ${ }^{43}$. Data was extracted manually from the Mascot report, and a minimum subset of proteins was assembled using only database entries for which unique peptide sequences were assigned.

Label-free quantification was performed using Skyline 2.5.0.5675 ${ }^{44}$ based on the database search results exported in Mascot .DAT format. Quantified peak areas provided by Skyline were used to aggregate cumulative peak areas of the top 3 or top 5 identified peptides ${ }^{45,46}$.

\section{Atomic model building}

The excellent experimental cryo-EM density allowed us to manually build an almost complete atomic model of the 39S subunit (Supplementary Table 1) using $\mathrm{O}^{47,48}$ (proteins) and COOT $^{49}$ (rRNA). The atomic model was mostly built based on our previous lower resolution structure ${ }^{6}$ or by complete de novo tracing of novel protein folds. $\mathrm{mL} 40, \mathrm{~mL} 46$, and $\mathrm{mL} 50$ were rebuilt based on templates from the yeast $54 \mathrm{~S}$ subunit structure ${ }^{5}$. We used the sequences of the isoforms identified by LC-MS/MS analysis (Supplementary Table 2) whenever a conclusive assignment could be reached. Three unassigned $\beta-$ strands were identified at a similar location to bL31m of the yeast $54 \mathrm{~S}$ structure. A genome-wide BackPhyre search ${ }^{50}$ using the corresponding yeast structure in the four implemented metazoan genomes (human, mouse, Drosophila melanogaster, and Caenorhabditis elegans) identified the mammalian mitoribosomal protein MRPL55 as the closest homolog of bL31m, although with relatively low sequence conservation $(<22 \%)$. Consequently, we renamed the MRPL55 to bL31m (see below) and corroborated the identity of the protein in the EM density. Remaining unoccupied EM density peaks could be interpreted as coordinated magnesium or zinc ions (zinc finger proteins bL32m, bL36m, and mL66 (MRPS18A), the latter forming a mixed zinc finger together with $\mathrm{uL} 10 \mathrm{~m}$, which contributes one zinc-coordinating residue). In spite of the high quality of our cryo-EM density, we cannot fully exclude register shifts in poorly ordered or peripheral regions of the structure. 


\section{Structure refinement and validation}

For the use of atomic coordinate refinement, the final 395 subunit cryo-EM maps from the automask output of RELION were clipped to a unit cell that generously accommodated the entire large subunit (resulting in arbitrary unit cell dimensions of $302.4 \AA \times 302.4 \AA \times 302.4 \AA$ with $\left.\alpha=\beta=\gamma=90^{\circ}\right)$. We then refined and validated the structure against reciprocal space data calculated from these cryo-EM maps (see below). The final refinement statistics are given in Extended Data Fig. 3a, and the distribution of B-factors in the refined molecular model is shown in Extended Data Fig. 3b.

For the conversion of the cryo-EM maps to reciprocal space structure factors we used a customized CNS ${ }^{51}$ input script, which blurs the phase probabilities of the structure factors in a resolution dependent manner based on the FSC values to account for the decreasing reliability of the map-derived amplitudes and phases with increasing resolution. For each of the resulting 1472132 reflections, a figure of merit (FOM, indicating the probability of the phase angle $\varphi$ to be correct) was calculated at the corresponding resolution ${ }^{52}$ according to equations (1) for the final map calculated from the full dataset and equation (2) for the half set maps calculated using data half sets ${ }^{53}$.

$\mathrm{FOM}=(2 \mathrm{FSC} /(1+\mathrm{FSC}))^{1 / 2}$

$\mathrm{FOM}=\mathrm{FSC}^{1 / 2}$

For the reciprocal space refinement in PHENIX ${ }^{54}$, experimental phase restraints were applied throughout by refining against the MLHL (phased maximum likelihood) target function after conversion of the FOMs and phase angles to the corresponding Hendrickson-Lattman (HL) coefficients, which represent an alternative way of expressing the phase probabilities. After one round of rigid body refinement (one rigid group for each protein and tRNA, five groups for the 16S rRNA), the entire model was refined to convergence by applying six macrocycles of individual coordinate and B-factor refinement using protein Ramachandran and secondary structure or RNA base-pair 
restraints to maintain good geometry in less well resolved regions of the map. In order to account for the specific electron scattering properties, we used the atomic electron scattering table provided by PHENIX.

Overfitting of the resulting model was monitored and minimized by a procedure according to the method described by ${ }^{5,35}$. In short, we manually tested different geometry weights during the refinement of the model against the full dataset. Models with reasonable geometry values $(90 \%$ of representative entries of similar resolution: root mean square deviation (r.m.s.d.) bonds $<0.014$, r.m.s.d. angles $<2.0$ deg., R-factors $<30 \%$ ) were further validated ${ }^{55}$. The coordinates of the models were displaced randomly by $0.1 \AA$ to remove potential model bias and subsequently re-refined to convergence against reciprocal space data generated from one of the half maps used in the final reconstruction. Then we calculated the FSC of the resulting model against each of the two half maps (Extended Data Fig. 3c-e) and compared the decay of the two FSC curves. A substantial gap between the two curves is indicative of overfitting ${ }^{5,35}$.

In our refinement we determined a weight of 1.0 for the reciprocal space data as the optimal balance between the overall fit to the experimental map and model geometry for our model (Extended Data Fig. 3c). Increasing the weight of reciprocal data during refinement leads to better agreement of the model to the half-map and lower R-factors but also leads to overfitting, indicated by higher FSC values for the half map used for refinement compared to the second half map, and worse model geometry (Extended Data Fig. 3d). Conversely, higher weighing of model geometry leads to a poorer fit to the map, reflected in higher R-factors and a lower FSC against the final map, but also reduces overfitting and results in a better model geometry (Extended Data Fig. 3e).

\section{Nomenclature of ribosomal proteins}

A new scheme for naming of ribosomal proteins was recently proposed ${ }^{10}$ and adopted by the field. We are using this new nomenclature system for naming 
of mitoribosomal proteins. For all mitoribosomal proteins with homologs in yeast mitoribosomes or in bacterial ribosomes, names have been established 5,10 . For mitoribosomal proteins without homologs in yeast, we use names that are as similar to the old naming system as possible (e.g. MRPL39 is now termed mL39). However, for mitoribosomal proteins MRPL57, MRPL58 (ICT1), and MRPL59 (CRIF1), this was not possible, as the corresponding $\mathrm{mLXX}$ names have been assigned to non-homologous yeast proteins ${ }^{5}$. We therefore use mL62 (MRPL58, ICT1), mL63 (MRPL57, MRP63), and mL64 (MRPL59, CRIF1) for these proteins (Supplementary Table 1). Further, as concluded from the BackPhyre ${ }^{50}$ searches mentioned above, MRPL55 is the mitoribosomal homolog of bacterial bL31 and has been designated bL31m.

\section{Creation of figures}

Figures depicting molecular structures were created using the UCSF Chimera package from the Computer Graphics Laboratory, University of California, San Francisco (supported by NIH P41 RR-01081) ${ }^{56}$ and PyMol (The PyMOL Molecular Graphics System, Version 1.7 Schrödinger, LLC.). Local resolution plots were generated in ResMap ${ }^{57}$.

\section{Online-only references}

31 Tivol, W. F., Briegel, A. \& Jensen, G. J. An improved cryogen for plunge freezing. Microsc. Microanal. 14, 375-379 (2008).

32 Mindell, J. A. \& Grigorieff, N. Accurate determination of local defocus and specimen tilt in electron microscopy. J. Struct. Biol. 142, 334-347 (2003).

33 Ludtke, S. J., Baldwin, P. R. \& Chiu, W. EMAN: semiautomated software for high-resolution single-particle reconstructions. J. Struct. Biol. 128, 82-97 (1999).

34 Scheres, S. H. W. \& Chen, S. Prevention of overfitting in cryo-EM structure determination. Nat. Meth. 9, 853-854 (2012).

35 Fernandez, I. S., Bai, X.-C., Murshudov, G., Scheres, S. H. W. \& Ramakrishnan, $V$. Initiation of translation by cricket paralysis virus IRES requires its translocation in the ribosome. Cell 157, 823-831 (2014).

36 Leitner, A. et al. Chemical cross-linking/mass spectrometry targeting acidic residues in proteins and protein complexes. Proc. Natl. Acad. Sci. USA (2014). 
37 Leitner, A. et al. Expanding the chemical cross-linking toolbox by the use of multiple proteases and enrichment by size exclusion chromatography. Mol. Cell. Proteomics 11, M111.014126 (2012).

38 Leitner, A., Walzthoeni, T. \& Aebersold, R. Lysine-specific chemical cross-linking of protein complexes and identification of cross-linking sites using LC-MS/MS and the XQuest/xProphet software pipeline. Nat. Protoc. 9, 120-137 (2014).

39 Walzthoeni, T. et al. False discovery rate estimation for cross-linked peptides identified by mass spectrometry. Nat. Meth. 9, 901-903 (2012).

40 Altschul, S. F., Gish, W., Miller, W., Myers, E. W. \& Lipman, D. Basic local alignment search tool. J. Mol. Biol. 215, 403-410 (1990).

41 Deutsch, E. W. et al. A guided tour of the Trans-Proteomic Pipeline. Proteomics 10, 1150-1159 (2010).

42 Perkins, D. N., Pappin, D. J., Creasy, D. M. \& Cottrell, J. S. Probabilitybased protein identification by searching sequence databases using mass spectrometry data. Electrophoresis 20, 3551-3567 (1999).

43 Koskinen, V. R., Emery, P. A., Creasy, D. M. \& Cottrell, J. S. Hierarchical clustering of shotgun proteomics data. Mol. Cell.

Proteomics 10, M110.003822 (2011).

44 Schilling, B. et al. Platform-independent and label-free quantitation of proteomic data using MS1 extracted ion chromatograms in skyline: application to protein acetylation and phosphorylation. Mol. Cell. Proteomics 11, 202-214 (2012).

45 Silva, J. C., Gorenstein, M. V., Li, G.-Z., Vissers, J. P. C. \& Geromanos, S. J. Absolute quantification of proteins by LCMSE: a virtue of parallel MS acquisition. Mol. Cell Proteomics 5, 144-156 (2006).

46 Fabre, B. et al. Comparison of label-free quantification methods for the determination of protein complexes subunits stoichiometry. EUPROT 4, 82-86 (2014).

47 Jones, T. A. Interactive electron-density map interpretation: from INTER to O. Acta Crystallogr. D Biol. Crystallogr. 60, 2115-2125 (2004).

48 Jones, T. A., Zou, J. Y., Cowan, S. W. \& Kjeldgaard, M. Improved methods for building protein models in electron density maps and the location of errors in these models. Acta Crystallogr. A Found. Crystallogr. 47 (Pt 2), 110-119 (1991).

49 Emsley, P., Lohkamp, B., Scott, W. G. \& Cowtan, K. Features and development of Coot. Acta. Crystallogr. D Biol. Crystallogr. 66, 486-501 (2010).

50 Kelley, L. A. \& Sternberg, M. J. E. Protein structure prediction on the Web: a case study using the Phyre server. Nat. Protoc. 4, 363-371 (2009).

51 Brunger, A. T. et al. Crystallography \& NMR system: A new software suite for macromolecular structure determination. Acta Crystallogr. D Biol. Crystallogr. 54, 905-921 (1998).

52 Read, R. J. Improved Fourier coefficients for maps using phases from partial structures with errors. Acta Crystallogr. A Found. Crystallogr. 42, 140-149 (1986). 
53 Rosenthal, P. B. \& Henderson, R. Optimal determination of particle orientation, absolute hand, and contrast loss in single-particle electron cryomicroscopy. J. Mol. Biol. 333, 721-745 (2003).

54 Adams, P. D. et al. PHENIX: a comprehensive Python-based system for macromolecular structure solution. Acta. Crystallogr. D Biol. Crystallogr. 66, 213-221 (2010).

55 Urzhumtseva, L., Afonine, P. V. \& Adams, P. D. Crystallographic model quality at a glance. Acta Crystallogr. D Biol. Crystallogr. 65, 207-300 (2009).

56 Pettersen, E. F. et al. UCSF Chimera--a visualization system for exploratory research and analysis. J Comput. Chem. 25, 1605-1612 (2004).

57 Kucukelbir, A., Sigworth, F. J. \& Tagare, H. D. Quantifying the local resolution of cryo-EM density maps. Nat. Meth. 11, 63-65 (2014).

58 Davydov, I. I. et al. Evolution of the protein stoichiometry in the L12 stalk of bacterial and organellar ribosomes. Nat. Commun. 4, 1387 (2013). 


\section{Figure Legends}

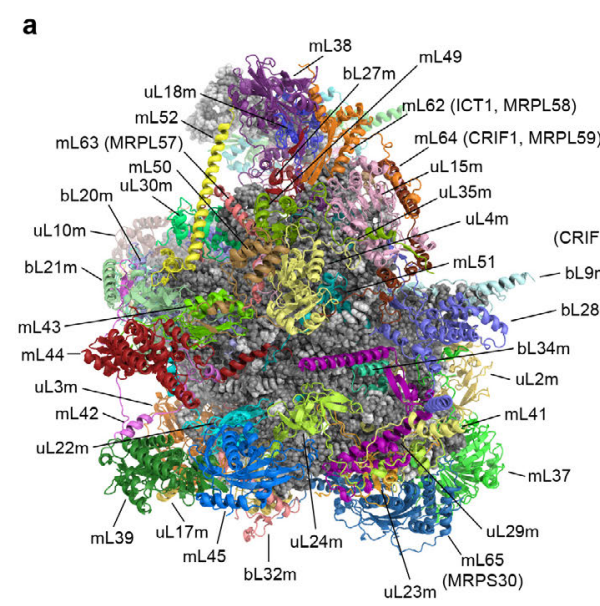

C

b
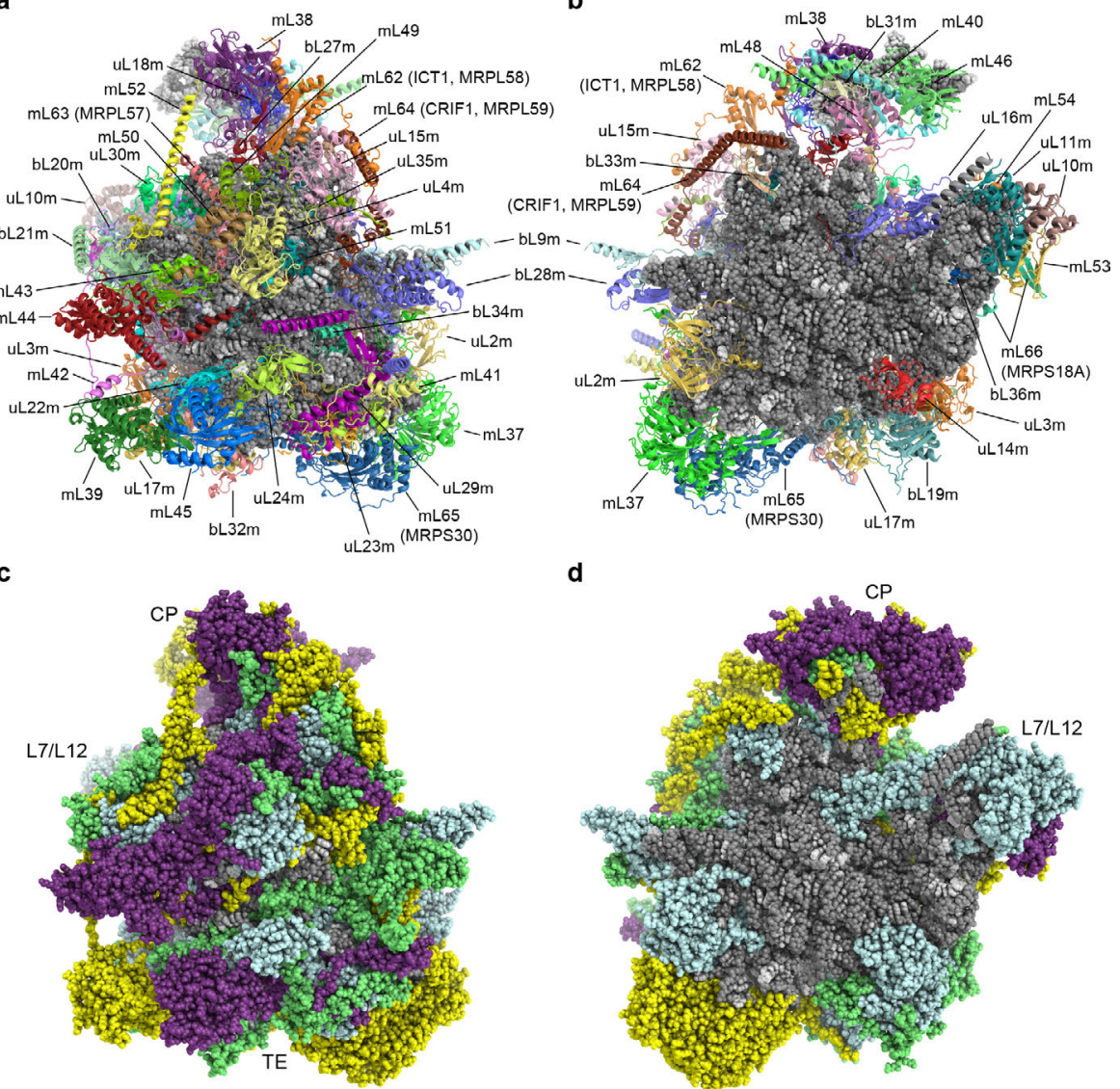

d

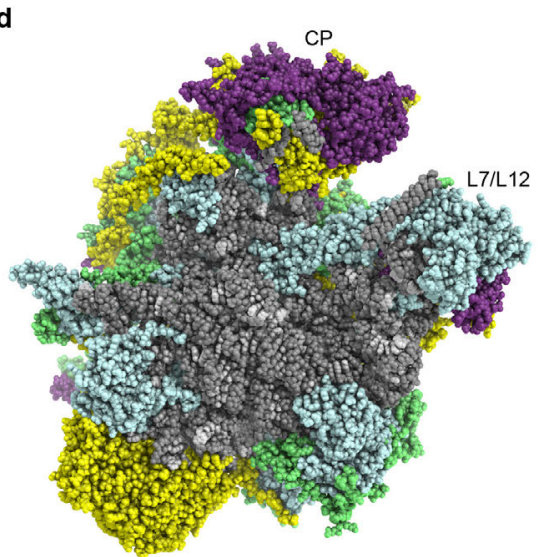

Figure 1 | Overall structure of the 395 subunit. a, b, Arrangement of proteins and rRNAs in the 39S subunit (left: solvent side; right, subunit interface side). c, d, 395 subunit (orientation as above) colored according to the conservation of rRNAs and ribosomal proteins. Grey: 16S rRNA and unassigned protein segments; cyan: proteins conserved in bacteria, mitoribosomal extensions in lime green; purple: proteins shared between yeast and mammalian mitoribosomes; yellow: CP tRNA and proteins specific to mitoribosomes of higher eukaryotes. Landmarks: CP, central protuberance; L7/L12, L7/L12 stalk base; TE, tunnel exit. 


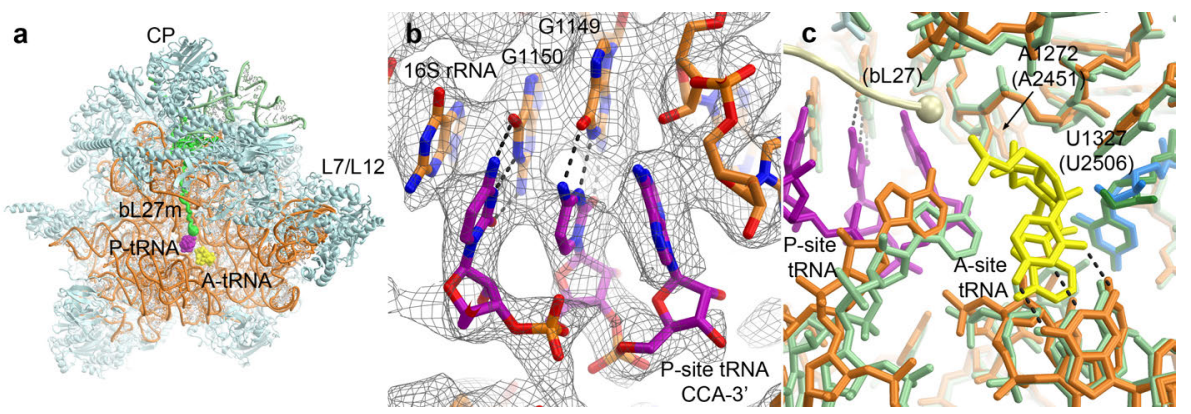

Figure 2 | The peptidyl transferase center of the 395 subunit. a, View into the PTC of the $39 \mathrm{~S}$ subunit. 16S rRNA in orange, mitoribosomal proteins in cyan, A- and P-site tRNA CCA-3' ends in yellow and purple, respectively, bL27m in bright green. $\mathbf{b}$, View of the CCA-3' end of the P-site tRNA bound near the PTC. Dashed lines indicate base pairing. c, Comparison of the bacterial ${ }^{15}$ and mitoribosomal PTCs (bacterial structural elements labeled in brackets). Colors as in a, with bacterial rRNA in green, bL27 in pale yellow, and base U1327 in dark blue (bacterial U1506 in dark green). 


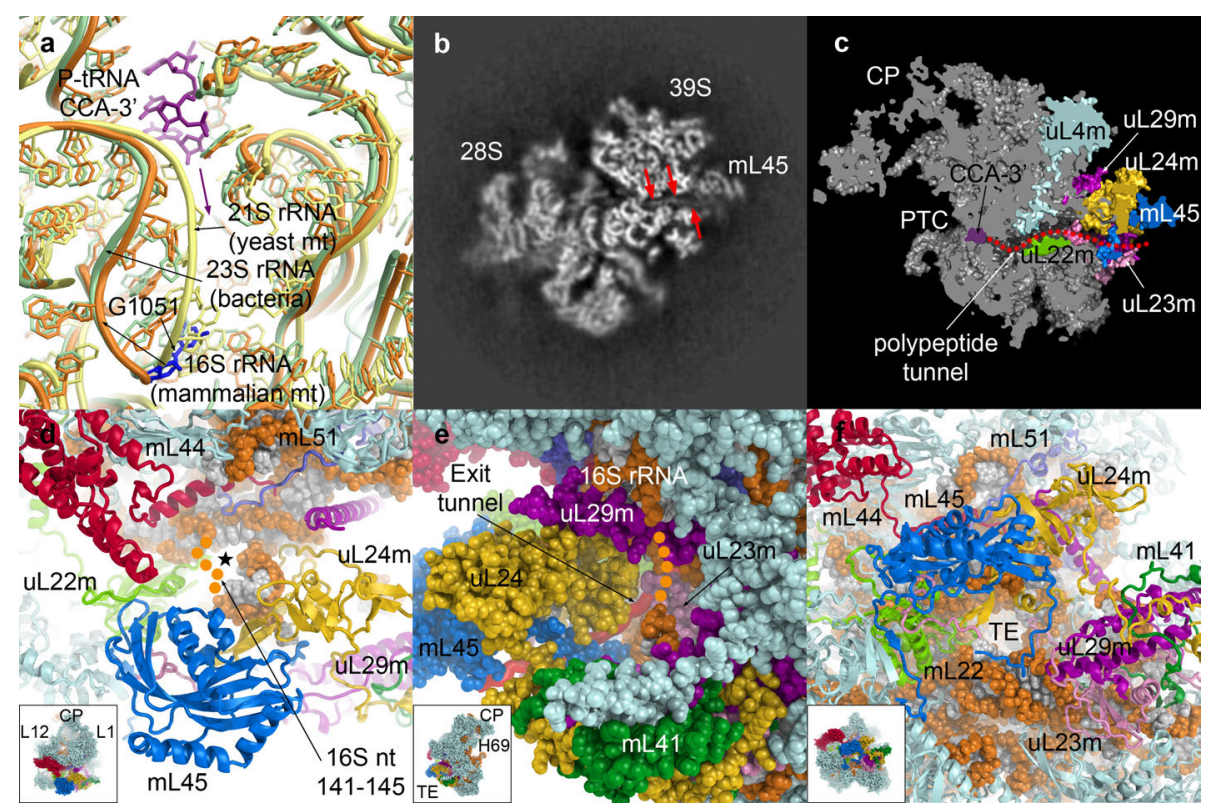

Figure 3 | The mitoribosomal polypeptide exit tunnel. a, Comparison of the tunnel entry in mammalian (orange; G1051 dark blue) and yeast (yellow) mitoribosomes and bacterial ribosomes (light green) ${ }^{5,15}$. P-site tRNA and tunnel entry (arrow) in purple. b, Slice through the 39S subunit cryo-EM map (filtered to $5.5 \AA$ resolution). A threadlike density inside the tunnel (red arrows) likely corresponds to the nascent polypeptide. c, Schematic representation of the exit tunnel path (same orientation as in b). $\mathbf{d}, 16 \mathrm{~S}$ rRNA nucleotides 141145 (indicated by orange dotted line because of low local resolution) block the proposed yeast polypeptide tunnel exit site (star) ${ }^{5}$. e, View of the polypeptide accessible site (PAS) ${ }^{3}$, which is reduced to a narrow opening by surrounding proteins and 16S rRNA nt 19-24 (not built, schematically indicated by a dotted line). Exit tunnel path indicated by a red tube. $\mathbf{f}$, View of the canonical polypeptide tunnel exit site (TE) and surrounding proteins. 


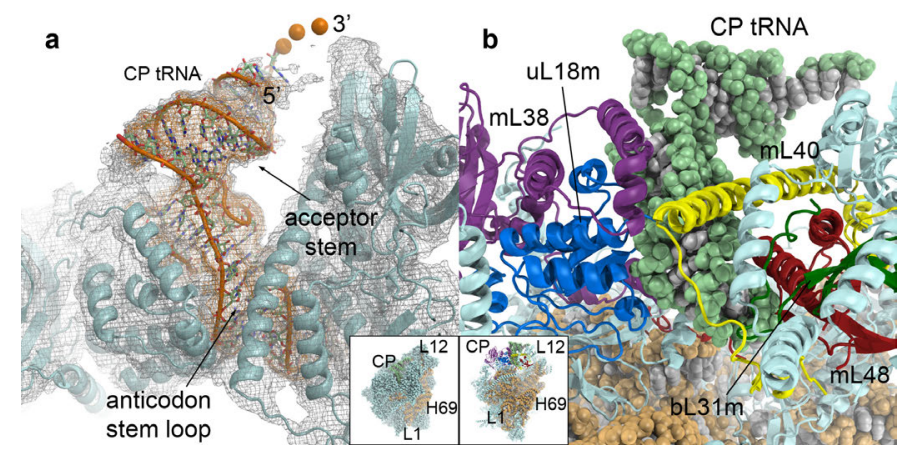

Figure 4 | The CP tRNA in the 39S subunit. a, The density for the CP tRNA (orange, map filtered to $6.5 \AA$ resolution) exhibits the L-shape characteristic of tRNAs (mitoribosomal proteins cyan; CP tRNA CCA-3' end lacking density shown as spheres). b. The CP tRNA (light green) forms extensive contacts to uL18m (blue), bL31m (dark green), mL38 (violet), mL40 (yellow), and mL48 (dark red). 


\section{Extended Data Figure Legends}

a

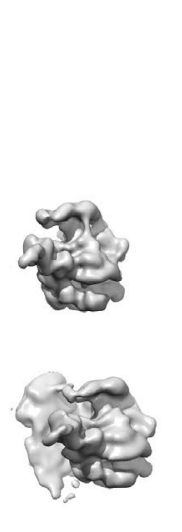

395 subunits bad particles

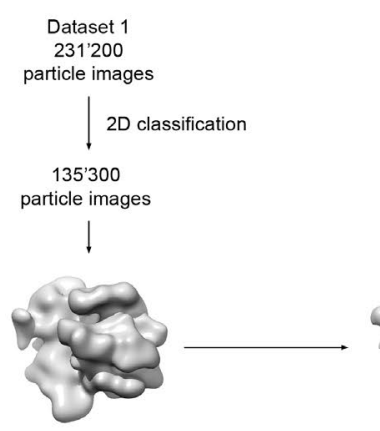

3D classification

(initial reference shown)
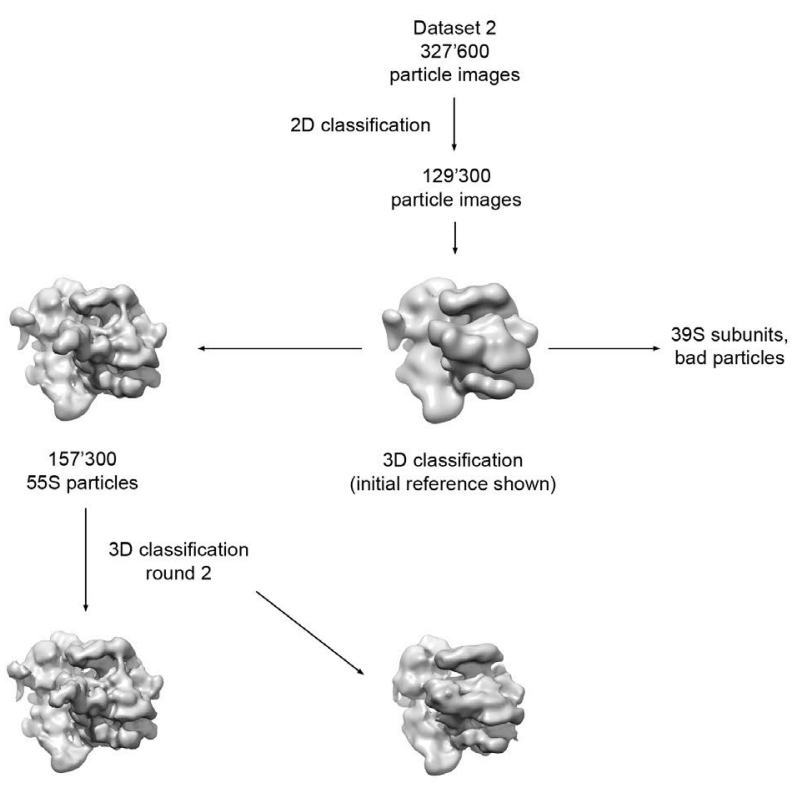

$141^{\prime} 700$

55 s particles

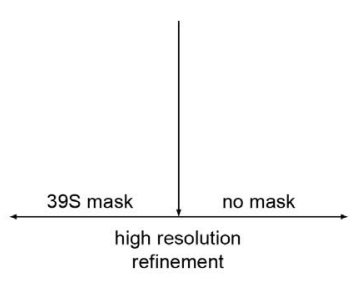

39 s subunit 3.4 A resolution
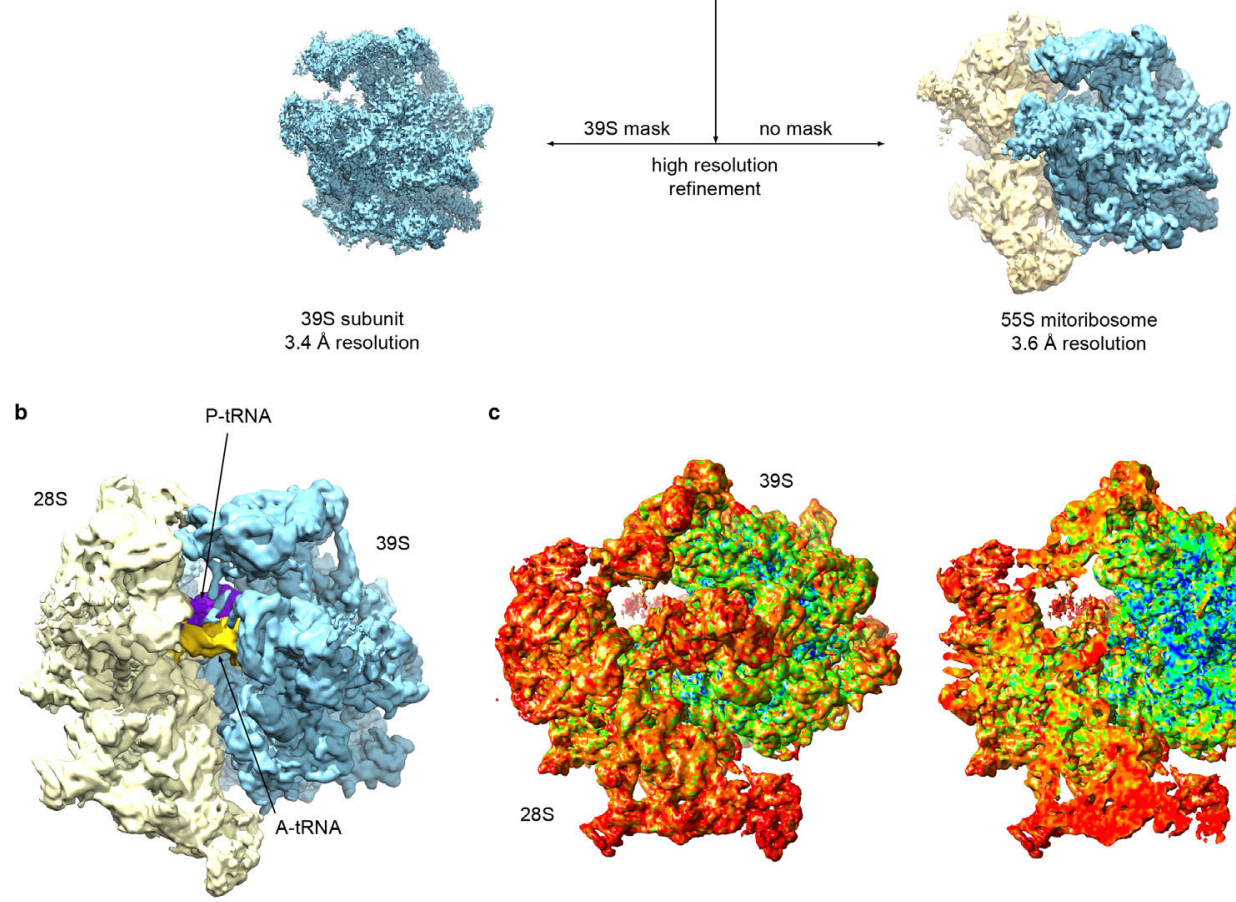

3.6 A resolution

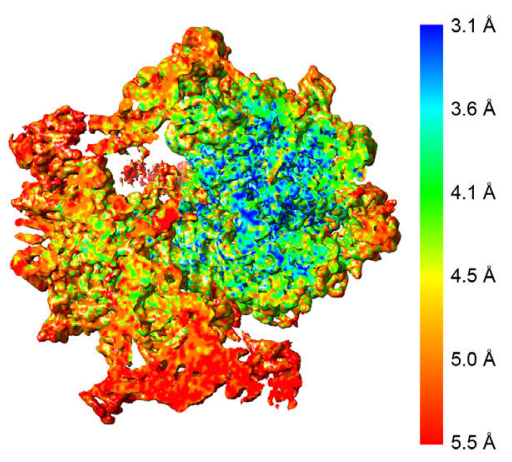

Extended Data Figure 1 | Computational sorting of the $55 \mathrm{~S}$ mitoribosome

datasets. a, In order to obtain a particle sub-population suitable for highresolution structure determination, two datasets were computationally sorted in RELION ${ }^{9} .2 \mathrm{D}$ and 3D classifications (only representative classes are shown) of a dataset of a total of roughly 560'000 particle images resulted in the selection of 141'700 55S mitoribosomal particles. The 55S particles were 
refined to obtain a reconstruction of the $55 \mathrm{~S}$ mitoribosome (3.6 $\AA$ resolution). b. The map obtained for the mammalian $55 \mathrm{~S}$ mitoribosome (28S subunit in pale yellow, 39S subunit in light blue) filtered to $6 \AA$ resolution. Two tRNA molecules are seen in the intersubunit space (A-site tRNA in yellow, P-site tRNA in purple). $\mathbf{c}$, Local resolution plot for the $55 \mathrm{~S}$ reconstruction (surface of the map on the left, viewed in section at the right). The local resolution is clearly below the average of $3.6 \AA$ in some part of the map, particularly in the $28 S$ subunit (4.1 $\AA$ on average for the $28 \mathrm{~S}$ subunit). Refinement with a mask around the 39S subunit (see a and Extended Data Fig. 2) resulted in an improved map suitable for de novo model building in the $39 \mathrm{~S}$ section of the map (3.4 ^ resolution). 

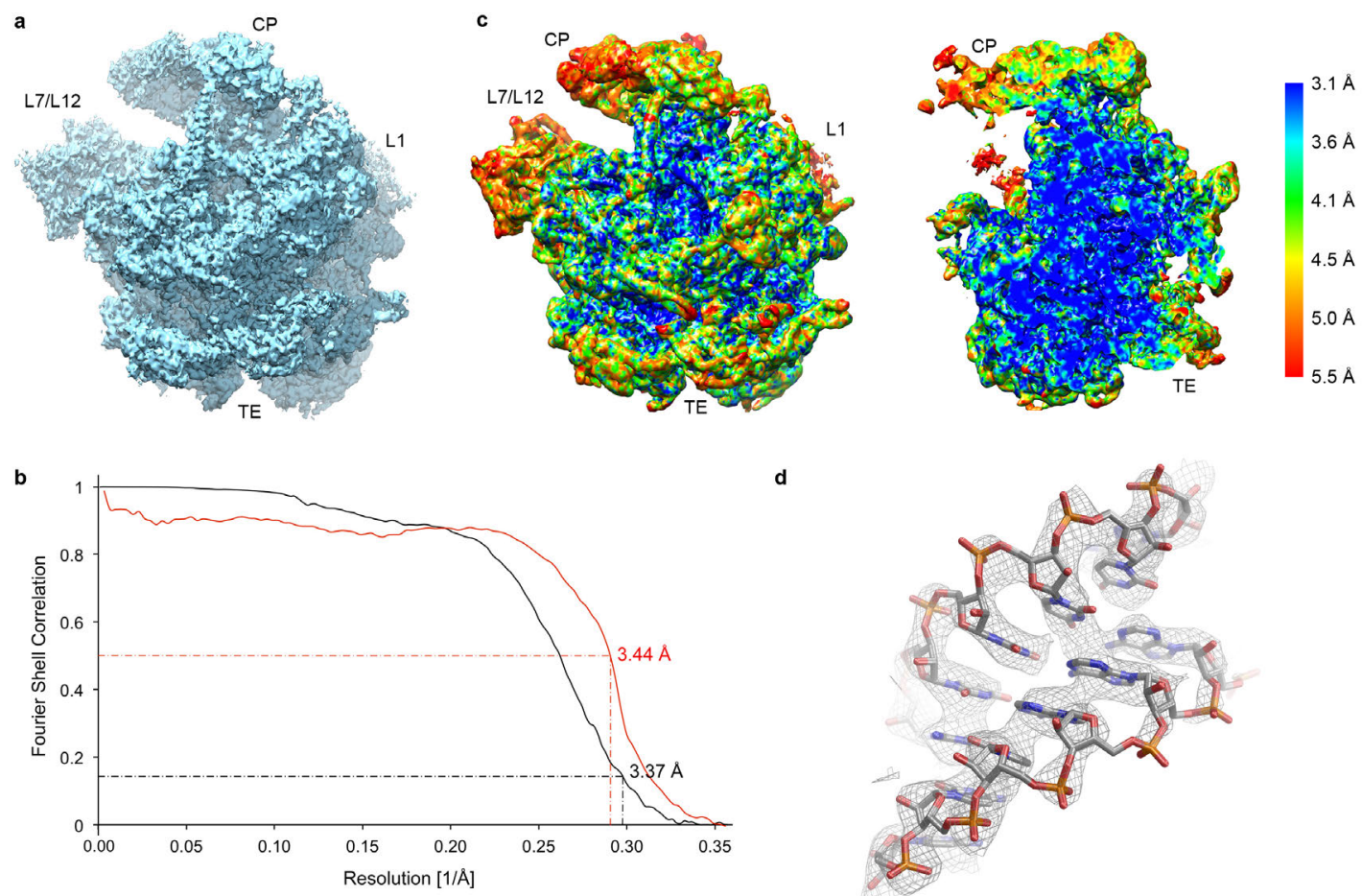

d
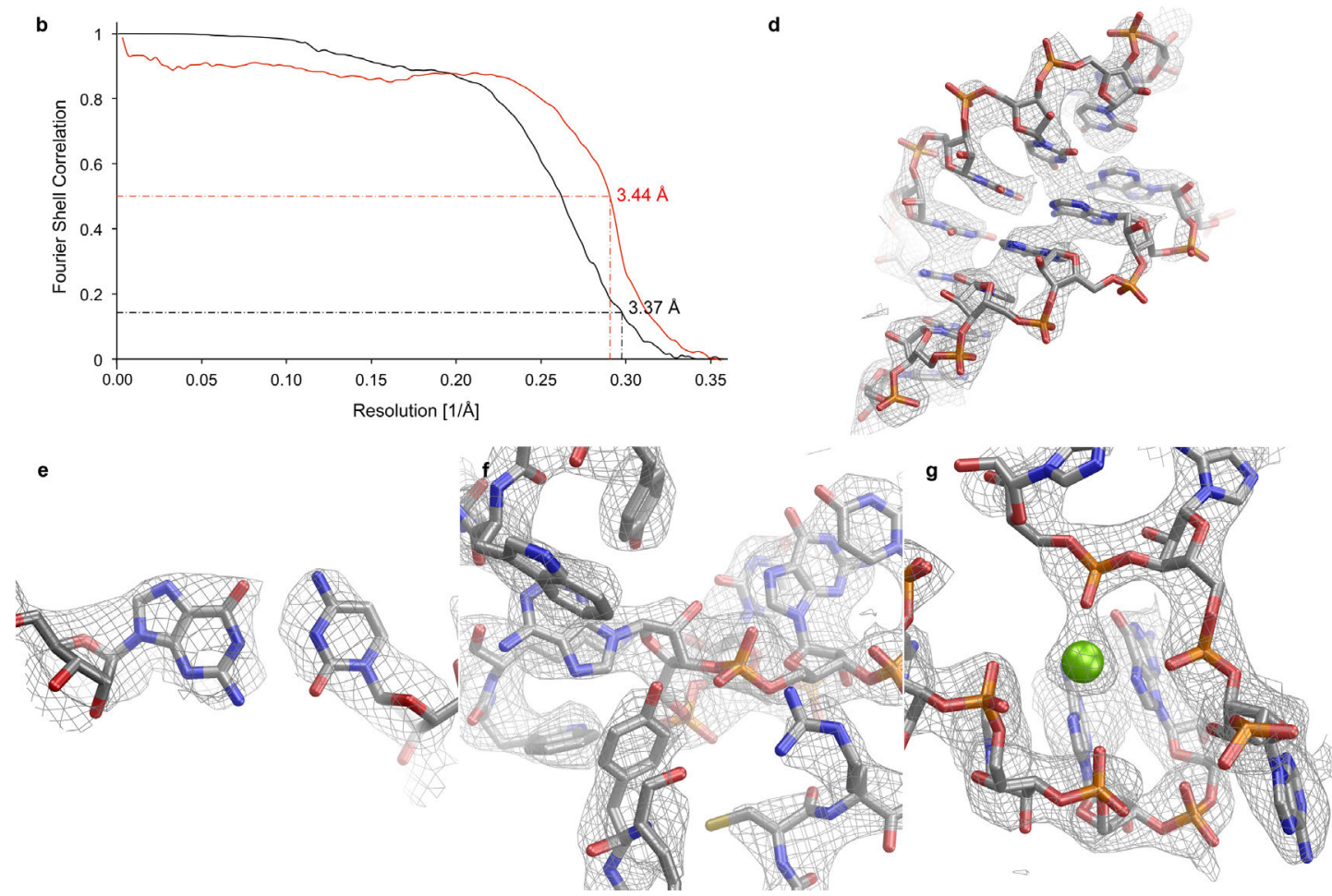

Extended Data Figure 2 | The cryo-EM map of the 395 subunit within the $55 S$ mitoribosome. a, Surface rendering of the 39S subunit cryo-EM map at $3.4 \AA$ resolution, viewed from the solvent side. Landmarks are indicated: $\mathrm{CP}$, central protuberance; L1, L1 stalk; L7/L12, L7/L12 stalk base; TE, polypeptide tunnel exit region. b, Black: FSC curve of the 39S cryo-EM reconstruction. The resolution is $3.4 \AA$ according to the $\mathrm{FSC}=0.143$ criterion ${ }^{53}$. Curve computed using RELION ${ }^{9}$ from auto-masked maps computed from data halfsets. Red: The FSC curve computed between the final reconstruction and the refined coordinates (see Extended Data Fig. 3) indicates a resolution of $3.4 \AA$ according to the FSC $=0.5$ criterion, which has to be used in this case ${ }^{53}$. c, Local resolution plot. $\mathbf{d}, \mathbf{e}, \mathbf{f}, \mathbf{g}$, Examples for the quality of the density: An rRNA double helix (d), a base pair (e), interactions of protein and rRNA with 
many large side chains visible (f), and a $\mathrm{Mg}^{2+}$ ion coordinated by the rRNA (g). 

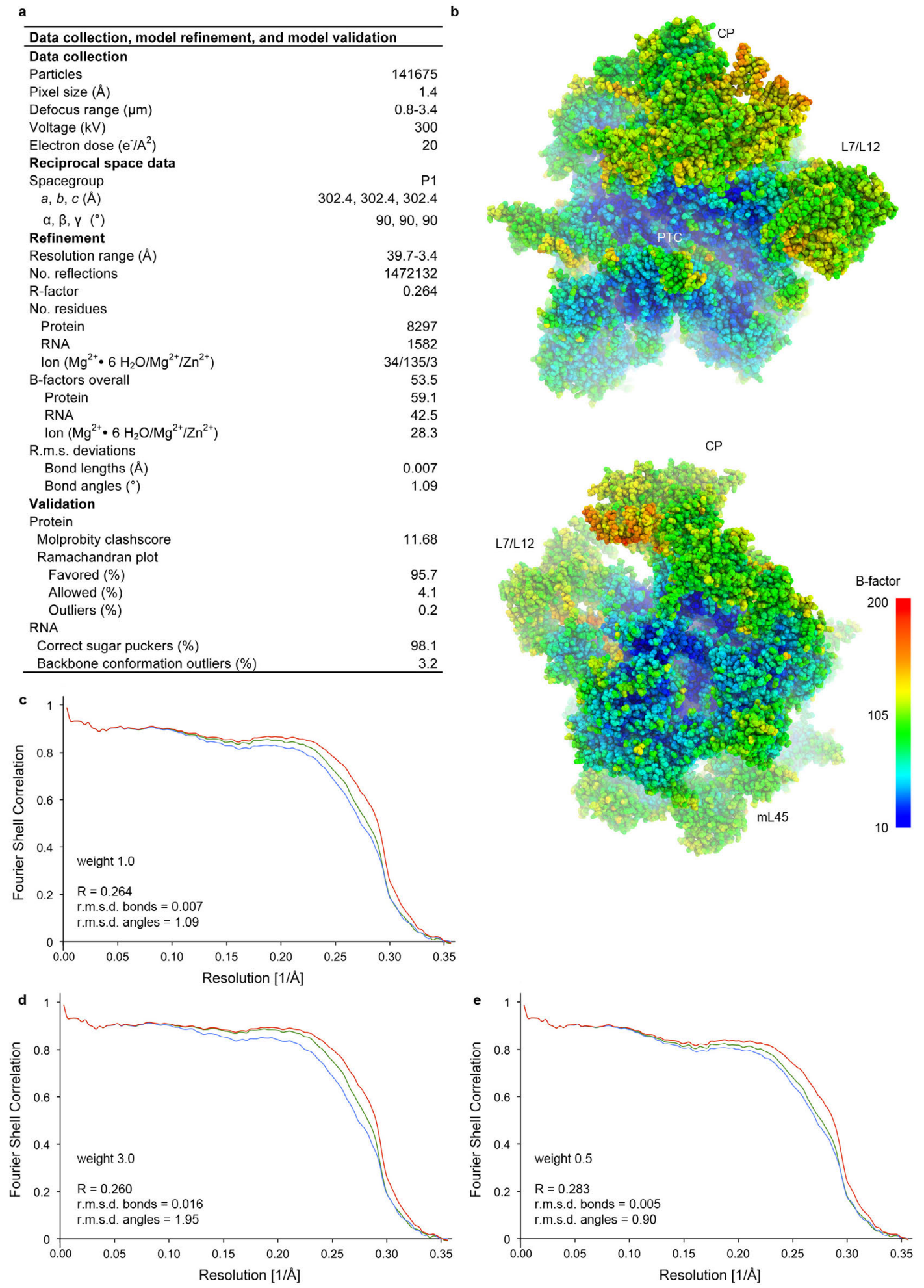

\section{Extended Data Figure 3 | Coordinate refinement of the 39S subunit}

atomic model. a, Model refinement and validation statistics. $\mathbf{b}$, Distribution of B-factors in the atomic model of the 39S subunit. Higher B-factors at the periphery, particularly in the $L 7 / L 12$ stalk and CP regions, reflect the lower local resolution in these areas (Extended Data Fig. 2c). c, Refinement weight 
optimization. FSC curves of the atomic coordinates refined into maps calculated from one half of the data (red: FSC model vs. map computed from the full dataset; blue: FSC model vs. half-set 1 (used for test refinement); green: model vs. half-set 2 (not used for test refinement)) using a weight of 1.0 for the reciprocal space data (see Methods for details). d, As in c, but with a weight of 3.0. e, As in c, but with a weight of 0.5 .

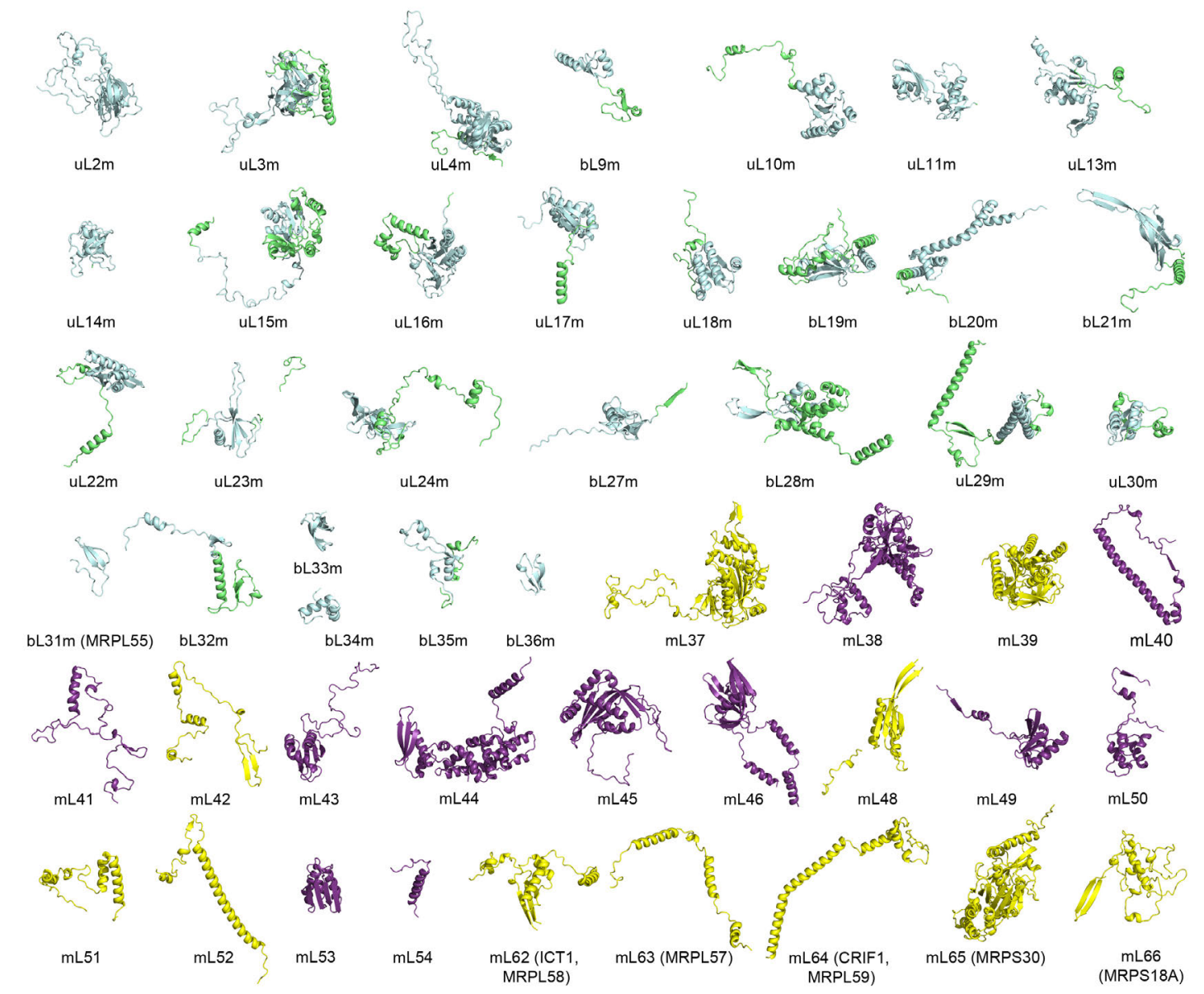

Extended Data Figure 4 | Overview of protein folds in the 395 subunit.

Overview of the structures of the 395 subunit proteins. Cyan: homologs of bacterial ribosomal proteins, with extensions in lime green; purple: mitochondrial-specific ribosomal proteins that are also present in yeast; yellow: mitoribosomal proteins without homologs in yeast. 


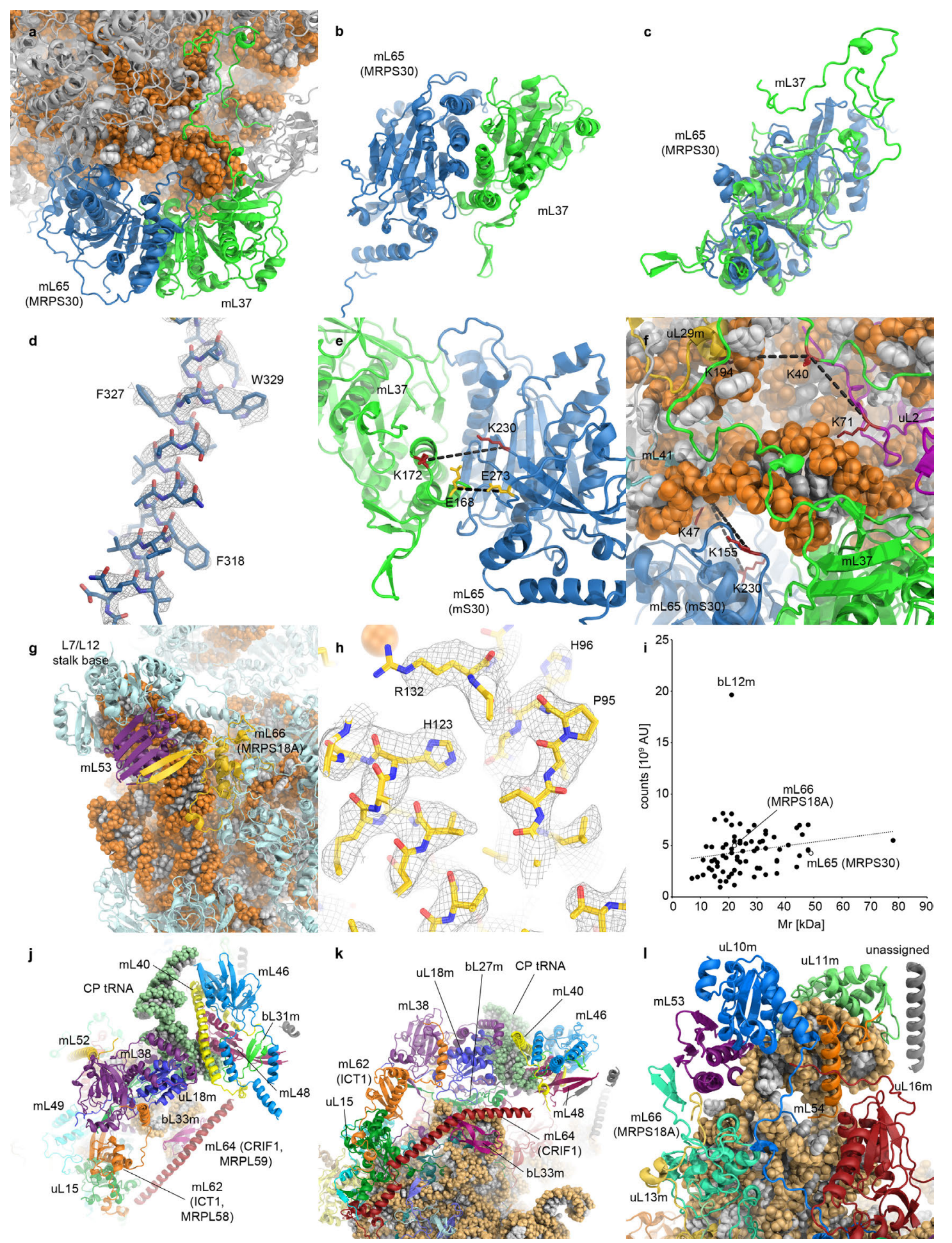

Extended Data Figure 5 | Protein structures in the 395 subunit. a, mL37

(green) and mL65 (MRPS30; blue) bound to the 39S subunit (16S rRNA backbone in orange, other mitoribosomal proteins in grey). b, mL37 and mL65 (MRPS30) form a pseudo-dimeric assembly on the 395 subunit. c, Superposition of mL37 and mL65 (MRPS30), revealing their common core fold with additional extensions specific to each protein. $\mathbf{d}$, Segment of the mL65 (MRPS30) structure shown in the cryo-EM map (only density 
corresponding to the selected structural element is shown). Side chain densities can be clearly identified and allowed the identification of the register of the mL65 (MRPS30) sequence in the map. e, Two XL-MS crosslinks between mL65 (MRPS30) and mL37 confirm the placement of mL65 (MRPS30) on the 39S subunit (red: DSS crosslink between lysines; gold: PDH crosslink between carboxyl groups). f, Crosslinks with other mitoribosomal proteins. DSS crosslinks of mL37 with uL2m (purple) and uL29m (gold) and of mL65 (MRPS30) with mL41 (cyan) were observed. All crosslink Ca-Ca-distances are $22 \AA$ or shorter. g, Binding site of mL66 (MRPS18A) at the L7/L12 stalk base. mL66 (MRPS18A; gold) and neighboring $\mathrm{mL} 53$ (purple) form a joint $\beta$-sheet. $\mathbf{h}$, Side chain density allows the unambiguous assignment of the location and trace of mL66 (MRPS18A). i, Semi-quantitative MS analysis of protein abundance in the $55 S$ subunit. mL66 (MRPS18A) and mL65 (MRPS30) are present in roughly stoichiometric amounts with other 395 subunit proteins, indicating that only one copy is present per $55 \mathrm{~S}$ mitoribosome. Only bL $12 \mathrm{~m}$ was detected with clearly more than one copy per $55 \mathrm{~S}$ mitoribosome, as expected from the multimeric architecture of the L7/L12 stalk ${ }^{58}$. j, Top view of the CP. In addition to the mitochondrial-specific CP tRNA, several mitochondrial-specific proteins have been recruited to the strongly remodeled CP of the 395 subunit. $\mathbf{k}$, Side view of the CP. Due to the missing 5S rRNA, the CP is connected to the subunit main body exclusively by protein contacts. I, Structure of the L7/L12 stalk base. Mitochondrial-specific ribosomal proteins $\mathrm{mL} 53, \mathrm{~mL} 54$, and $\mathrm{mL} 66$ (MRPS18A) have been recruited to the L7/L12 stalk base and may stabilize its connection to the $39 \mathrm{~S}$ subunit main body. 


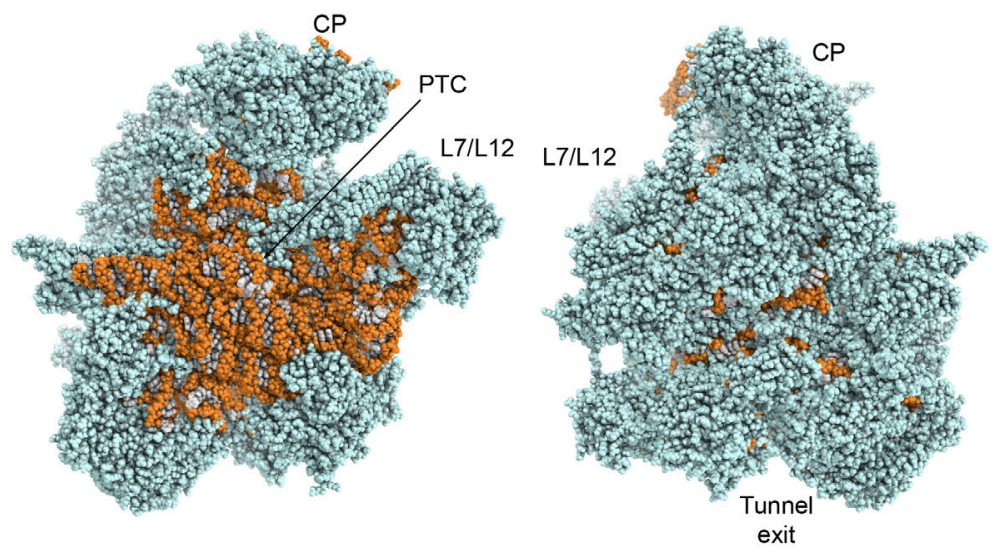

b

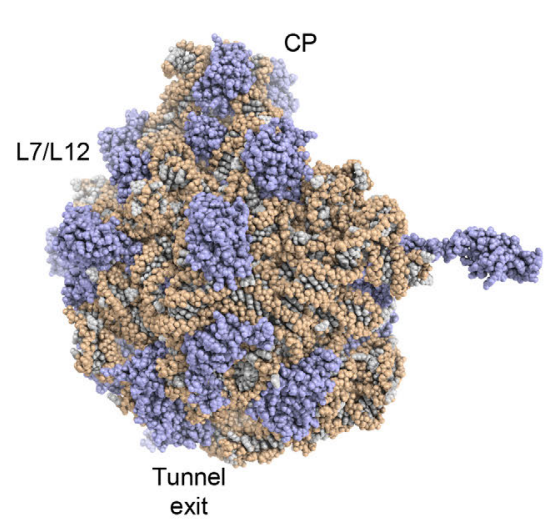

Extended Data Figure 6 | Overall distribution of rRNA and proteins in the mitochondrial $39 \mathrm{~S}$ subunit and the bacterial $50 \mathrm{~S}$ subunit. a, Mitochondrial ribosomal large subunit (subunit interface side on the left, solvent exposed side on the right). Proteins (cyan) almost entirely cover the solvent exposed side of the 395 subunit, leaving barely any rRNA (orange) exposed. b, In bacterial ribosomes ${ }^{15}$ (L1 stalk omitted for clarity), a significant portion of the ribosomal surface on the solvent exposed side is composed of rRNA (brown), with ribosomal proteins (slate blue) scattered across the subunit surface. The subunit interface side, which makes contact to the small subunit and binds tRNA substrates, is formed by rRNA to a large extent in both types of ribosomes. Landmarks are indicated: L7/L12, L7/L12 stalk base; CP, central protuberance; PTC, peptidyl transferase center; Tunnel exit, polypeptide tunnel exit site. 

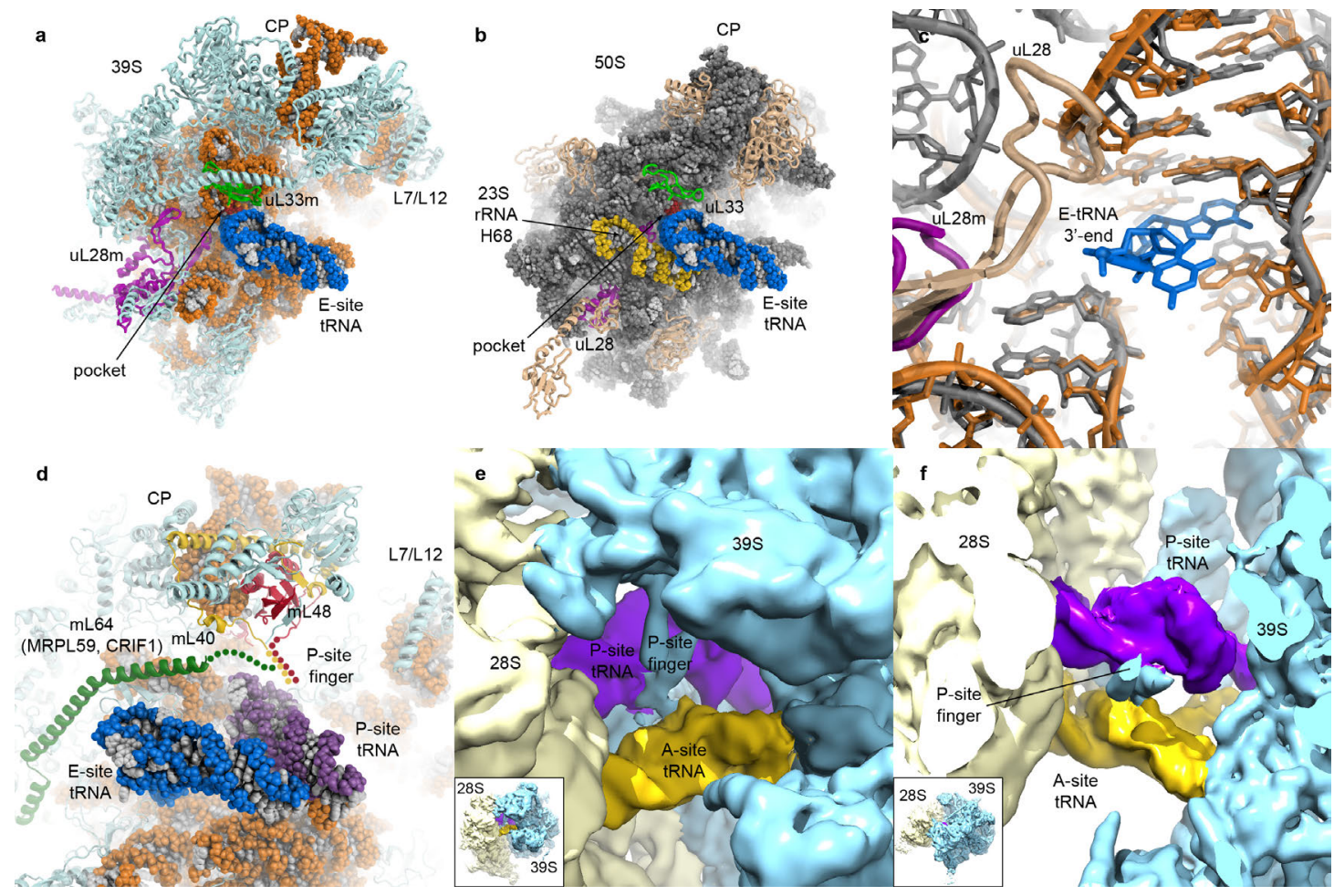

Extended Data Figure 7 | The E-site and the P-site finger of the 39S

subunit. a, 39S subunit (16S rRNA orange, ribosomal proteins cyan, uL28m purple, uL33m green) with E-site tRNA (blue) modeled based on the superposition of the structure of the bacterial ribosome with bound tRNAs ${ }^{15}$. The residues corresponding to the tRNA-3' end binding pocket in bacteria ${ }^{15}$ are shown in dark red. b, In bacterial ribosomes (23S rRNA grey, ribosomal proteins light brown, L1 stalk omitted for clarity, otherwise as in a), rRNA helix 68 (yellow) additionally interacts with the E-site tRNA. This structural element is missing in the 395 subunit. c, The pocket into which the terminal nucleotide of the E-site tRNA inserts in bacterial $23 \mathrm{~S}$ rRNA (grey) ${ }^{26}$ is conserved in the mitochondrial 16S rRNA (orange). Ribosomal protein uL28 (light brown) interacts with the E-site tRNA in bacteria ${ }^{15}$, but is shortened in the $39 \mathrm{~S}$ subunit (uL28m in purple). d, mL40 (yellow), mL64 (CRIF1, MRPL59) (green) and $\mathrm{mL} 48$ (red) approach the area where the E- and P-site tRNAs are located based on superposition of the bacterial structure ${ }^{15}$. 16S rRNA in orange, 39S proteins in pale cyan. e, Cryo-EM density of the $55 \mathrm{~S}$ mitoribosome (filtered to $6 \AA$ resolution) segmented into $39 \mathrm{~S}$ and $28 \mathrm{~S}$ subunits and A- and P-site tRNAs. The P-site finger is inserted between the A- and P-site tRNAs and contacts both tRNAs. $\mathbf{f}$, Same as e, but shown in top view with part of the ribosome segmented away to reveal the tRNAs. 


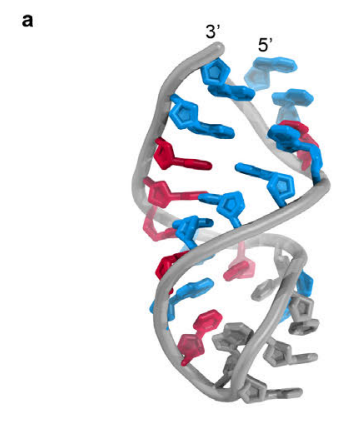

5'-RRYRRRRYRYNNNNRNYRYYYRR-3
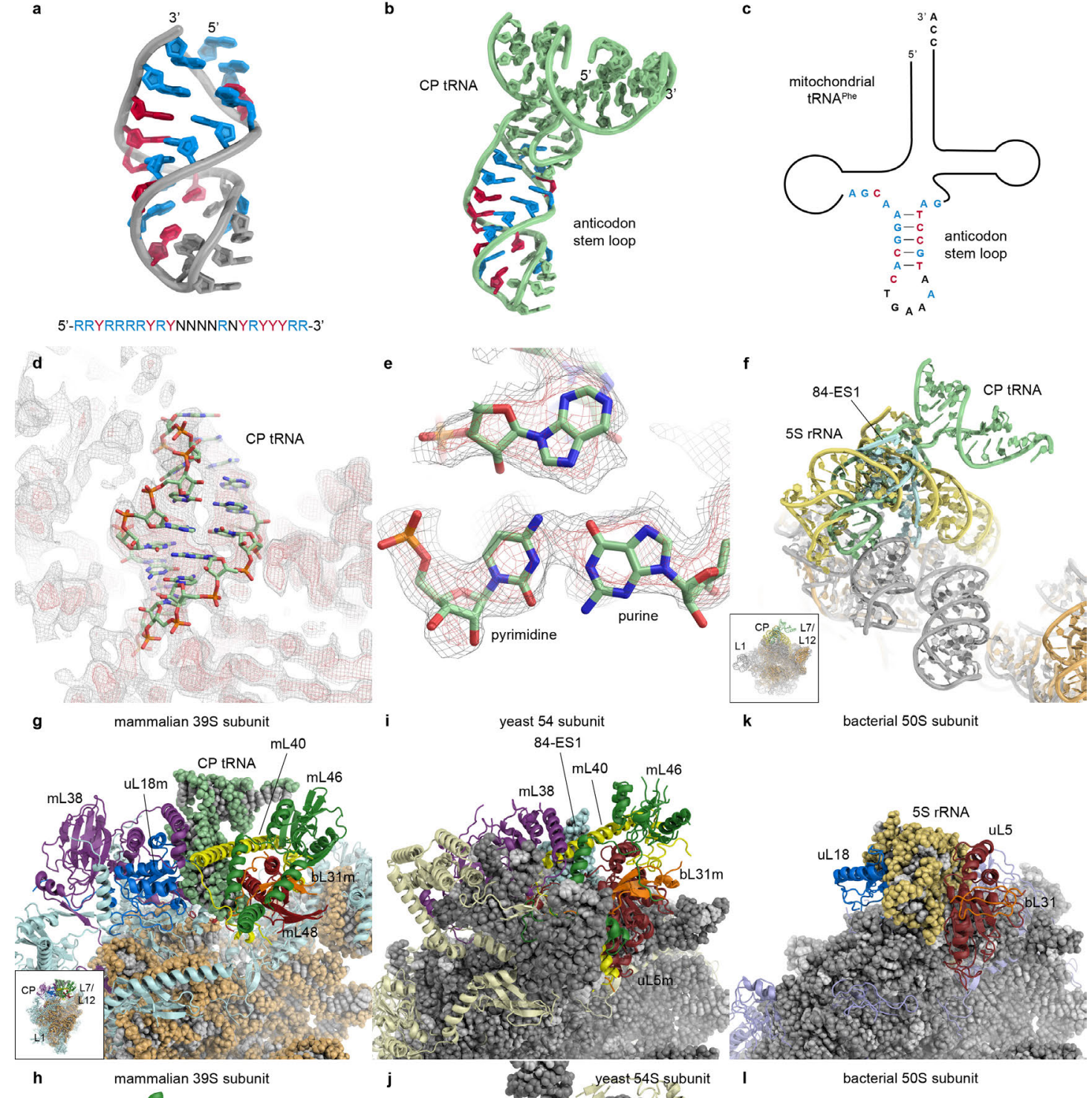

$c$
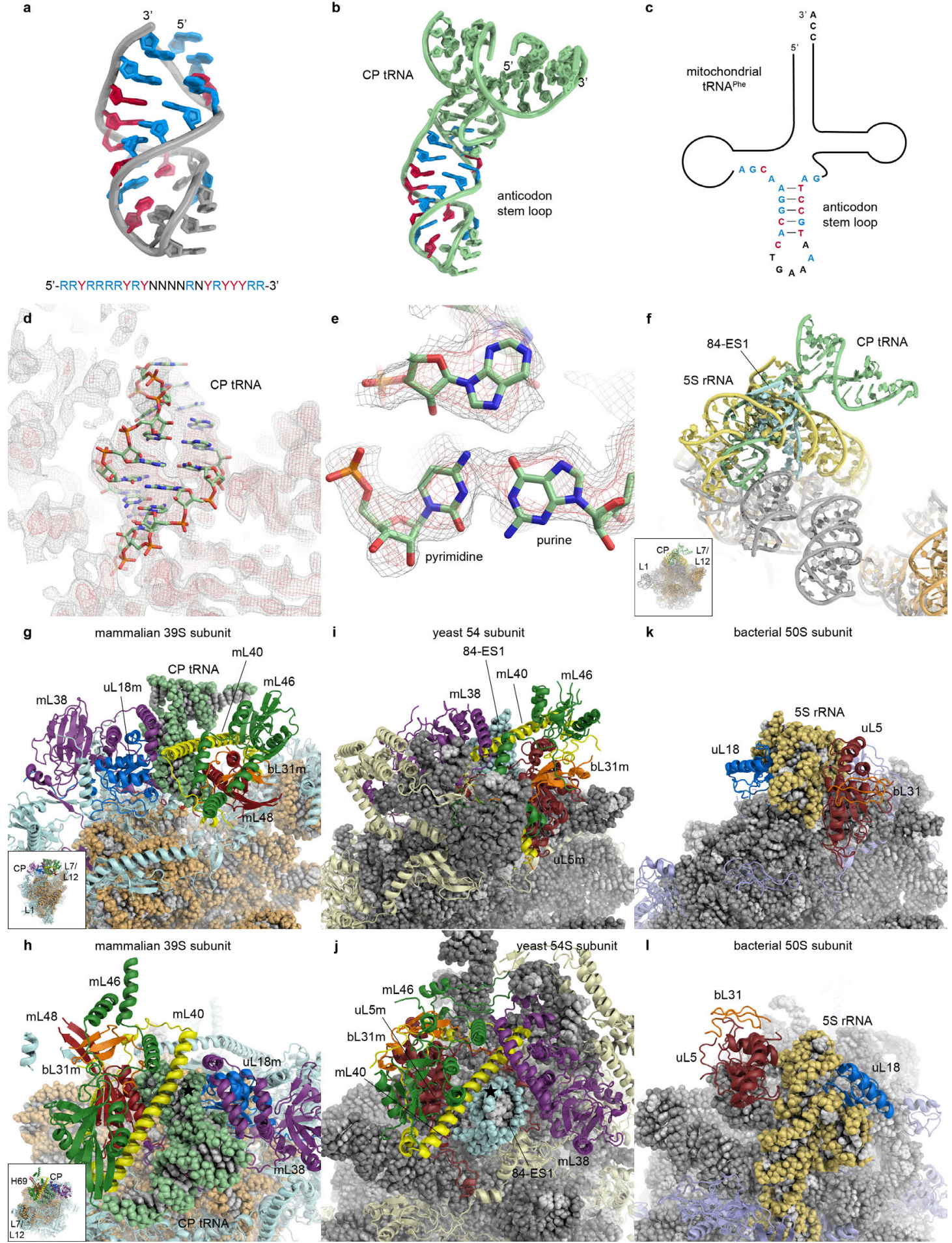

\section{Extended Data Figure 8 | The architecture of the mammalian and yeast}

mitoribosomal central protuberances. a, The purine-pyrimidine pattern observed in the additional RNA density at the CP as built model (top) and sequence (bottom). Y, pyrimidines; R, purines; $N$ undetermined. $\mathbf{b}$, The model of CP tRNA with bases in the segment corresponding to the pattern in a colored accordingly. c, Simplified secondary structure diagram of mitochondrial tRNA ${ }^{\mathrm{Phe}}$ with the segment corresponding to the pattern in a 
colored accordingly. $\mathbf{d}$, View of the CP tRNA and the cryo-EM density in the vicinity. Phosphate positions are clearly visible, but the stacked bases merge into continuous strands due to lower local resolution. e, Purines and pyrimidines can be distinguished by their shape. $\mathbf{f}$, Superposition of the large subunit rRNAs of bacterial ${ }^{15}$ (23S rRNA in grey, $5 S$ rRNA in yellow), mammalian mitochondrial (16S rRNA in orange, CP tRNA in light green), and yeast mitochondrial ribosomes ${ }^{5}$ (only 84-ES1 shown for clarity, cyan). $\mathbf{g}, \mathbf{h}$, Side- and top views of the mammalian mitoribosomal CP with the CP tRNA in light green, $\mathrm{mL} 38$ in purple, $\mathrm{uL} 18 \mathrm{~m}$ in blue, $\mathrm{mL} 40$ in yellow, $\mathrm{mL} 46$ in dark green, $\mathrm{mL} 48$ in red, and bL31 $\mathrm{m}$ in orange. $\mathbf{i}, \mathbf{j}$, Side- and top views of the yeast mitoribosomal $\mathrm{CP}{ }^{5}$ with $\mathrm{mL} 38$ in purple, $\mathrm{mL} 40$ in yellow, $\mathrm{mL} 46$ in dark green, bL31m in orange, uL5m in dark red, and 84-ES1 in cyan. Homologous proteins in yeast and mammals occupy roughly similar positions, while mammalian $\mathrm{mL} 48$ is positioned similarly to yeast $\mathrm{UL} 5 \mathrm{~m}$. Mammalian $\mathrm{mL} 38$ is shifted slightly towards the solvent side relative to its yeast counterpart. 84ES1 occupies a similar position as the anticodon stem-loop part of mammalian CP tRNA, and $\mathrm{mL} 40$ binds to the RNA minor groove (star) in both cases. $\mathbf{k}, \mathbf{l}$, View of the bacterial ribosomal $\mathrm{CP}^{15}$. $23 \mathrm{~S}$ rRNA in light grey, $5 \mathrm{~S}$ rRNA in yellow, uL18 in blue, uL5 in dark red, bL31 in orange, other ribosomal proteins in light blue. 

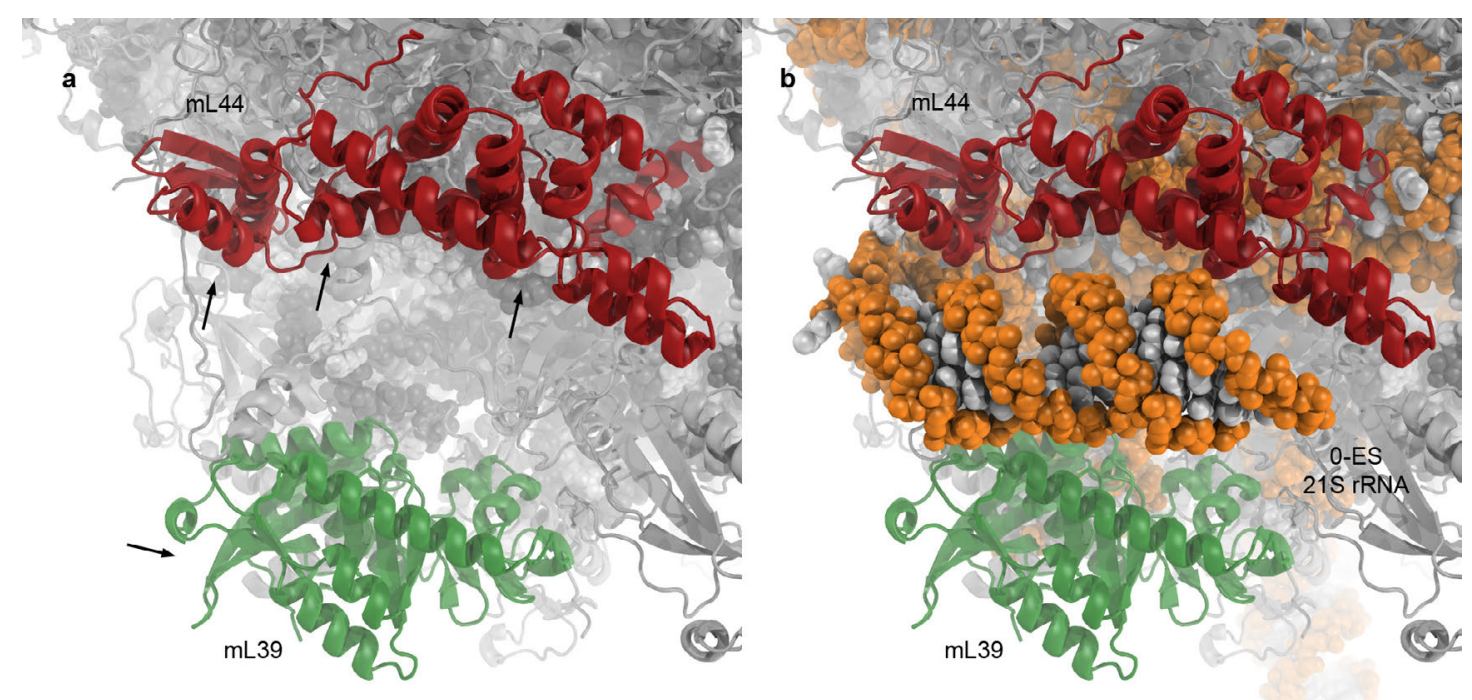

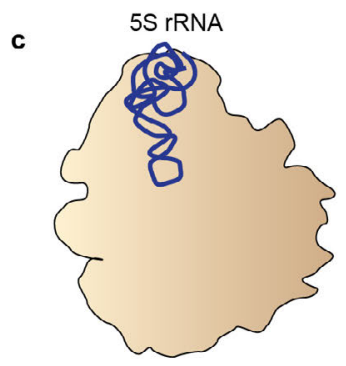

bacterial

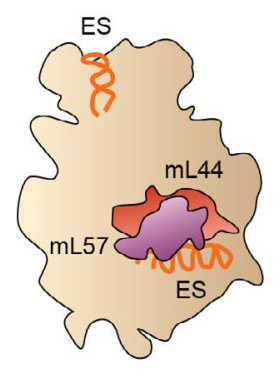

yeast
mitochondrial

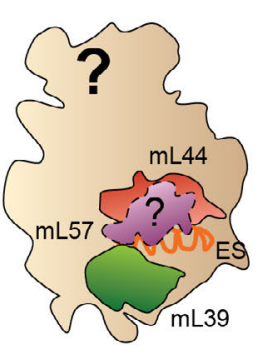

intermediate

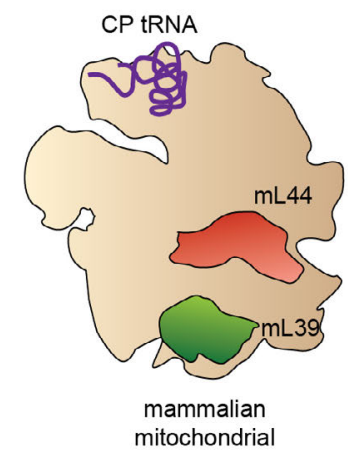

mitochondrial

\section{Extended Data Figure 9 | Evolution of RNA binding mitochondrial}

ribosomal proteins. a, In the mammalian mitoribosome, the surfaces of mL39 (green) and mL44 (red) corresponding to the RNA binding surfaces of their RNA binding homologs (arrows) are exposed to the solvent. $\mathbf{b}$, The presence of an rRNA expansion segment in the yeast mitoribosome (yeast $21 \mathrm{~S}$ rRNA ${ }^{5}$ in orange superimposed on the $39 \mathrm{~S}$ subunit) explains the arrangement of proteins observed in the mammalian mitoribosome. c, Schematic of the evolution of this region of the mitoribosome. Before the loss of the rRNA ESs, $\mathrm{mL} 39, \mathrm{~mL} 44$, and $\mathrm{mL} 57$ were possibly simultaneously present in evolutionary precursors of mammalian mitoribosomes. 


\section{Extended Data Table 1 | CX-MS crosslinks used for confirmation of}

protein localizations. Peptides: crosslinked peptides including location of the crosslinked residues in the peptides; Protein/residue: protein name and residue number of crosslinked amino acids; $M_{r}$ : theoretical molecular mass of the crosslink product; $\mathrm{m} / \mathrm{z}$ : experimentally determined mass-to-charge ratio; Id score: identification score calculated by xQuest.

\begin{tabular}{|c|c|c|c|c|c|}
\hline Peptides & $\begin{array}{l}\text { Proteinl } \\
\text { residue }\end{array}$ & $\begin{array}{l}\text { Protein/ } \\
\text { residue }\end{array}$ & $\begin{array}{l}M_{r} \\
\text { (Da) }\end{array}$ & $\mathrm{m} / \mathrm{z}$ & $\begin{array}{l}\text { Id } \\
\text { score }\end{array}$ \\
\hline \multicolumn{6}{|c|}{ DSS } \\
\hline $\begin{array}{l}\text { KPEPPPLDR-TKYTVMPVK-a1- } \\
\text { b2 }\end{array}$ & $\begin{array}{c}\mathrm{mL} 37 \\
\mathrm{~K} 40\end{array}$ & $\begin{array}{l}\mathrm{uL} 2 \mathrm{~m} \\
\mathrm{~K} 71\end{array}$ & 2251.223 & 751.416 & 31.98 \\
\hline $\begin{array}{l}\text { YQINDKPHNQIR-DGKFVQIK- } \\
\text { a6-b3 }\end{array}$ & $\begin{array}{l}\mathrm{mL} 65 \\
\mathrm{~K} 230\end{array}$ & $\begin{array}{c}\mathrm{mL} 41 \\
\mathrm{~K} 47\end{array}$ & 2596.369 & 866.464 & 29.84 \\
\hline $\begin{array}{l}\text { YQINDKPHNQIR- } \\
\text { IWHSTEDIPKR-a6-h10 }\end{array}$ & $\mathrm{mL} 65$ & $\begin{array}{l}\mathrm{mL} 37 \\
\mathrm{~K} 172\end{array}$ & 3043.556 & 761.897 & 27.78 \\
\hline $\begin{array}{l}\text { QWPIPWYLNKK-KPEPPPLDR- } \\
\text { a10-b1 }\end{array}$ & $\begin{array}{l}\mathrm{uL} 29 \mathrm{~m} \\
\mathrm{~K} 194\end{array}$ & $\begin{array}{l}\mathrm{mL} 37 \\
\mathrm{~K} 40\end{array}$ & 2657.432 & 665.366 & 27.06 \\
\hline KRAPVIQDR-DGKFVQIK-a1-b3 & $\begin{array}{l}\mathrm{mL} 65 \\
\mathrm{~K} 155\end{array}$ & $\begin{array}{c}\mathrm{mL} 41 \\
\mathrm{~K} 47\end{array}$ & 2153.232 & 539.316 & 26.63 \\
\hline \multicolumn{6}{|c|}{ PDH } \\
\hline $\begin{array}{l}\text { QYENTIFIGSK-LWHSTEDIPK- } \\
\text { a3-b6 }\end{array}$ & $\begin{array}{l}\mathrm{mL} 65 \\
\mathrm{E} 273\end{array}$ & $\begin{array}{l}\mathrm{mL} 37 \\
\mathrm{E} 168\end{array}$ & 2675.37 & 669.85 & 25.92 \\
\hline
\end{tabular}

